\title{
Dynamics of dissolved inorganic carbon in the Midriff Islands region of the Gulf of California: Influence of water masses
}

\section{Dinámica del carbono inorgánico disuelto en la región de las grandes islas del golfo de California: Influencia de las masas de agua}

\author{
José Martín Hernández-Ayón ${ }^{1 *}$, Cecilia Chapa-Balcorta ${ }^{1,2}$, Francisco Delgadillo-Hinojosa', \\ Víctor Froylan Camacho-Ibar ${ }^{1}$, Miguel Angel Huerta-Díaz', Eduardo Santamaría-del-Angel ${ }^{2}$, \\ Salvador Galindo-Bect ${ }^{1}$, José Antonio Segovia-Zavala ${ }^{1}$ \\ ${ }^{1}$ Instituto de Investigaciones Oceanológicas (IIO), Universidad Autónoma de Baja California, Apartado postal \\ 453, Ensenada, CP 22800, Baja California, México. \\ 2 Facultad de Ciencias Marinas, Universidad Autónoma de Baja California, Apartado postal 453, Ensenada, \\ CP 22800, Baja California, México.
}

* Corresponding author. E-mail: jmartin@uabc.edu.mx

\begin{abstract}
In the Midriff Islands region (MIR) of the Gulf of California (Mexico), tidal mixing plays a very important role in the distribution of chemical properties. Although the temporal variability of the water masses is linked to the ocean dynamics of this region, its importance has not been considered. This work estimates the effect of water mass dynamics on the spatial distribution and seasonal variation of dissolved inorganic carbon (DIC) in the region, with special emphasis on the Ballenas Channel, during winter 2002, spring 2003, and summer 2004. Gulf of California Water (GCW) was found throughout the area in the first $100 \mathrm{~m}$. Below, Subtropical Subsurface Water (SSW) filled the Delfín, Salsipuedes, and Tiburón basins, while to the southeast it was found between 150 and $350 \mathrm{~m}$. Pacific Intermediate Water did not cross the sills. SSW enriches the surface water of the MIR with DIC when it increases in volume and when mixed with GCW by tidal effects. This contribution decreases towards summertime, when SSW sinks and the volume of GCW increases. In winter, GCW had three water types associated with oceanographic mixing processes and in combination with the presence of SSW. There was a strong DIC vs temperature relationship, which can be applied to analyze the carbon dynamics in the MIR, both at the surface using satellite imagery and vertically to simulate physical processes.
\end{abstract}

Key words: dissolved inorganic carbon, Gulf of California, vertical mixing, Ballenas Channel, coastal DIC studies.

RESUMEN. En la región de las grandes islas (RGI) del golfo de California la mezcla por mareas juega un papel muy importante en la distribución de propiedades disueltas. Aunque la variabilidad temporal de las masas de agua está ligada con la dinámica oceanográfica de la región, su importancia no ha sido considerada. En este trabajo se estima el efecto de la dinámica de las masas de agua sobre la distribución espacial y variación estacional del carbono inorgánico disuelto (CID) en la RGI, con especial énfasis en el canal de Ballenas, durante el invierno de 2002, la primavera de 2003 y el verano de 2004. El Agua del Golfo de California (AGC) se encontró en toda la superficie en los primeros $100 \mathrm{~m}$ de profundidad. Por debajo del AGC, el Agua Subsuperficial Subtropical (ASS) llenó las cuencas Delfín, Salsipuedes y Tiburón, mientras que en el sureste se encontró entre 150 y 350 m. El Agua Intermedia del Pacifico no atravesó los umbrales. Se encontró que el ASS enriquece de CID las aguas superficiales de la RGI cuando aumenta su volumen y cuando se mezcla con el AGC por efecto de las mareas. El aporte disminuye hacia el verano, cuando el ASS se hunde al mismo tiempo que el volumen de AGC aumenta. En invierno, el AGC presentó tres tipos de agua asociadas a los procesos de mezcla y en combinación con la presencia de ASS. Se encontró también que en la RGI la relación CID vs temperatura podría aplicarse en futuros estudios para analizar la dinámica del carbono en la RGI, tanto en la superficie mediante imágenes de satélite como en la vertical para simular procesos físicos.

Palabras clave: carbono inorgánico disuelto, golfo de California, mezcla vertical, canal de Ballenas, estudios costeros de CID.

\section{INTRODUCTION}

Upwelled water generally has a high concentration of dissolved inorganic carbon (DIC). Hence, in upwelling regions, $\mathrm{pCO}_{2 \text { sea }}$ is greater than $\mathrm{pCO}_{2 \mathrm{~atm}}$ and favors a positive flux from the ocean to the atmosphere. In turn, if $\mathrm{CO}_{2}$ is consumed by phytoplankton, surface $\mathrm{pCO}_{2}$ can decrease and be less than $\mathrm{pCO}_{2 \mathrm{~atm}}$, resulting in a negative flux of $\mathrm{CO}_{2}$ from the atmosphere to the ocean. In the Midriff Islands region (MIR)

\section{INTRODUCCIÓN}

Generalmente, el agua que aflora a la superficie tiene una alta concentración de carbono inorgánico disuelto (CID). Por lo tanto, en regiones de surgencia, la $\mathrm{pCO}_{2 \operatorname{mar}}$ es mayor que la $\mathrm{pCO}_{2 \text { atm }}$ y favorece un flujo positivo del océano a la atmósfera. En cambio, si el $\mathrm{CO}_{2}$ es consumido por el fitoplancton, la $\mathrm{pCO}_{2}$ superficial puede disminuir y ser menor que la $\mathrm{pCO}_{2 \mathrm{~atm}}$, y se presentaría un flujo negativo de $\mathrm{CO}_{2}$ de 
of the Gulf of California (GC), the vertical transport of nutrients (Álvarez-Borrego et al. 1978, Lavín et al. 1995, Torres-Valdés 2000) and DIC (Gaxiola-Castro et al. 1978; Hernández-Ayón et al. 2007a, 2007b) is strongly controlled by tide-related mixing. Several studies have also reported a constant and tide-induced fornightly modulation of inorganic carbon and nutrient input in this area (Hidalgo-González et al. 1997, Martínez-Díaz-de-León et al. 2006), as well as effects due to the intense interaction between tidal currents and bathymetry (Marinone-Moschetto and Lavín-Peregrina 2003). For example, in summer, $80 \%$ of the distribution of cadmium (which has a nutrient-type vertical distribution), DIC, and $\mathrm{pH}$ in the MIR can be explained by vertical mixing (Delgadillo-Hinojosa et al. 2001, Hernández-Ayón et al. 2007a). In winter, Hernández-Ayón et al. (2007b) observed the formation of peculiar oceanographic regions resulting from the effect of intense mixing on carbon dynamics.

Another notable characteristic of the MIR is that even when nutrient concentrations are high, pigment concentrations are not (Santamaría-del-Angel et al. 1994), possibly because phytoplankton blooms do not occur in completely mixed waters (Falkowski and Raven 1997). Consistent with these observations, the highest concentrations of nutrients, but not of pigments, have been recorded in Ballenas Channel (Santamaría-del-Angel et al. 1994). An analysis of satellitederived pigment measurements in the MIR revealed that during spring-summer tides, chlorophyll concentrations were higher on the west coast and showed a southward displacement, whereas during winter-spring tides there was more spatial variability than in summer (Flores-de-Santiago et al. 2007).

These findings indicate that both physical processes and tidal mixing play an important role in the distribution of chemical properties. Although the temporal variability of the water masses is linked to the regional ocean dynamics, its importance has not been considered. A volumetric analysis of the water masses in the GC (Torres-Orozco 1993) revealed that Gulf of California Water (GCW), Subtropical Subsurface Water (SSW), and Pacific Intermediate Water (PIW) occupy most of the volume of the MIR. An analysis of the proportions of water masses in a latitudinal transect from the MIR to the mouth of the GC indicated $36 \% \mathrm{SSW}, 57 \% \mathrm{GCW}$, and $7 \%$ Equatorial Surface Water (ESW) in the first $50 \mathrm{~m}$ in summertime, whereas in wintertime, when ESW flows out of the GC (Álvarez-Borrego and Schwartzlose 1979), the proportions were $70 \%$ SSW and 30\% GCW (Delgadillo-Hinojosa 2000). Hernández-Ayón et al. (2007b) also observed that under winter conditions, SSW is located very close to the surface, between 50 and $75 \mathrm{~m}$ depth, and reported that SSW could be an important source of DIC in the region. Based on these observations, it is reasonable to assume that waters rich in $\mathrm{CO}_{2}$ will occur in the MIR during the cold season due to the larger proportion of SSW in the area.

Few studies have been conducted on the spatial distribution and seasonal variation of DIC in the GC, and further la atmósfera al océano. En la región de las grandes islas (RGI) del golfo de California (GC), el transporte vertical de nutrientes (Álvarez-Borrego et al. 1978, Lavín et al. 1995, Torres-Valdés 2000) y de CID (Gaxiola-Castro et al. 1978; Hernández-Ayón et al. 2007a, 2007b) está fuertemente controlado por procesos de mezcla asociados a la marea. Varios trabajos también han documentado que el aporte de carbono inorgánico y de nutrientes es constante y está modulado quincenalmente por las mareas (Hidalgo-González et al. 1997, Martínez-Díaz-de-León et al. 2006). Además, existe un efecto debido a la intensa interacción entre las corrientes de marea y la batimetría (Marinone-Moschetto y LavínPeregrina 2003). Por ejemplo, en un estudio efectuado en verano en la RGI, se registró que la distribución del cadmio (que tiene una distribución vertical tipo nutriente), CID y pH podría ser explicada en un $80 \%$ por la mezcla vertical (Delgadillo-Hinojosa et al. 2001, Hernández-Ayón et al. 2007a). En otro estudio realizado en invierno, HernándezAyón et al. (2007b) observaron una formación de regiones oceanográficas peculiares producto del efecto de la intensidad de la mezcla sobre la dinámica del carbono.

Otra característica notable de la RGI es que aun cuando las concentraciones de nutrientes son altas, las concentraciones de pigmentos no lo son (Santamaría-del-Angel et al. 1994), lo cual pudiera deberse a que los florecimientos de fitoplancton no ocurren en las aguas completamente mezcladas (Falkowski y Raven 1997). Consistente con estas observaciones, en el canal de Ballenas se han registrado las concentraciones más altas de nutrientes, pero no de pigmentos (Santamaría-del-Angel et al. 1994). En un análisis de datos satelitales de pigmentos durante diferentes condiciones de marea en la RGI se encontró que durante las mareas de primavera-verano, las concentraciones de clorofila eran más altas en la costa oeste y presentaron un desplazamiento hacia el sur, mientras que durante las mareas de invierno-primavera había más variabilidad espacial que durante el verano (Flores-de-Santiago et al. 2007).

Estos antecedentes señalan que tanto los procesos físicos como la mezcla por mareas juegan un papel muy importante en la distribución de propiedades disueltas. Aunque la variabilidad temporal de los volúmenes de las masas de agua está ligada con la dinámica oceanográfica de la RGI, su importancia no ha sido considerada. Un análisis volumétrico de las masas de agua del GC (Torres-Orozco 1993) indicó que el Agua del Golfo de California (AGC), el Agua Subsuperficial Subtropical (ASS) y el Agua Intermedia del Pacifico (AIP) ocupan el mayor volumen de agua de la RGI. Un análisis de las proporciones de las masas de agua en un transecto latitudinal de la RGI a la boca del GC indicó que durante el verano se explicó el $36 \%$ de ASS, $57 \%$ de AGC y $7 \%$ de Agua Superficial Ecuatorial (ASE) en los primeros $50 \mathrm{~m}$ superficiales, mientras que en invierno, al retraerse el ASE hacia el exterior del GC (Álvarez-Borrego y Schwartzlose 1979), las proporciones de mezcla fueron $70 \%$ de ASS y $30 \%$ de AGC (Delgadillo-Hinojosa 2000). Hernández-Ayón et al. (2007b) 
information of the processes controlling its variability is needed to subsequently be able to quantify the reservoirs. In this paper we report on the spatial distribution of DIC in a wide sampling grid in the MIR under winter conditions. We also assess the temporal variation of DIC in Ballenas Channel during three seasons and assess the effect of water mass dynamics on the chemical properties of the waters in this region of the GC.

\section{MATERIALS AND METHODS}

\section{Study area}

The GC is located on the west coast of Mexico, and is approximately $1100 \mathrm{~km}$ long and $150 \mathrm{~km}$ wide (fig. 1). The MIR bathymetry constitutes one of the most notable features of the GC (fig. 1b). It is formed by a series of deep basins and sills that, on the one hand, restrict the circulation between the northern and central GC and, on the other, act as a point where intense mixing is generated by strong tidal currents (Torres-Orozco 1993). In the southern and central GC, the depth of the basin is greater than $3000 \mathrm{~m}$ and there is unrestricted water exchange with the Pacific Ocean (Castro et al. 2000). In the southern part of the MIR, the San Esteban ( $\sim 600 \mathrm{~m}$ deep) and San Lorenzo ( 400 m deep) sills restrict the entry of water masses to Ballenas Channel $(\sim 1500 \mathrm{~m}$ deep). In the northern part of Ballenas Channel, there is another sill that connects with Delfín Basin ( $>800 \mathrm{~m}$ deep) (Badan-Dangon et al. 1991).

An oceanographic campaign, Umbrales I, was carried out in the MIR in March 2002 aboard the R/V Francisco de Ulloa, initiating during spring tides and ending during neap tides. The sampling grid covered 38 stations (fig. 1a) where CTD (Sea-Bird 911 Plus) casts were performed. At 26 of these stations, seawater samples for DIC analysis were collected with Niskin bottles at the surface and at different depths $(5,10,20,30,50,75,100,200,250,300,400,500$, $700,750,800,900$, and $1000 \mathrm{~m}$ ), depending on the bathymetry. Seawater was taken from the Niskin bottles with $50-\mathrm{mL}$ syringes and DIC was measured on board using the potentiometric titration technique described by Hernández-Ayón et al. (1999).

To analyze the temporal variation of the presence of water masses and their effect on DIC content, data collected in March 2002 (Umbrales I), May 2003 (Umbrales II), and September 2004 (Umbrales III) from the same depths at three representative stations in Ballenas Channel were compared (fig. 1a).

\section{Calibration of the Umbrales I DIC measurements}

The potentiometric DIC measurements from the Umbrales I cruise were calibrated with DIC measurements of deep-water samples collected at station 38 during the Umbrales II campaign in May 2003 and the Umbrales III también observaron que bajo condiciones de invierno, la ASS se localizó muy cerca de la superficie, entre los $50 \mathrm{~m}$ y $75 \mathrm{~m}$ de profundidad, y señalaron que dicha masa de agua podría ser una fuente muy importante de CID en esta región. Con base en estas observaciones, se esperaría encontrar aguas superficiales ricas en $\mathrm{CO}_{2}$ en la RGI durante la época fría del año debido a la mayor proporción de ASS en la zona.

Existen pocos estudios sobre la distribución espacial y la variabilidad temporal del CID en el GC, por lo que es necesario aumentar el conocimiento sobre los procesos que controlan la variabilidad de CID para cuantificar los reservorios. En este trabajo se registra la distribución espacial de CID en una amplia red de muestreo en la RGI bajo condiciones de invierno. Adicionalmente se analiza la variación estacional del CID en el canal de Ballenas durante tres épocas del año y se estima el efecto de la dinámica de las masas de agua sobre las propiedades químicas de las aguas en esta zona del GC.

\section{MATERIALES Y MÉTODOS}

\section{Área de estudio}

El GC se localiza en la costa oeste de México y mide aproximadamente $1100 \mathrm{~km}$ de largo y $150 \mathrm{~km}$ de ancho (fig. 1). La batimetría de la RGI (fig. 1b) constituye una de las características más sobresalientes del GC debido a que está conformada por una serie de umbrales y cuencas profundas que, por una parte, restringen la circulación entre el norte y centro del GC y, por otra, actúan como puntos de generación de mezcla intensa por fuertes corrientes de marea (Torres-Orozco 1993). En el sur y centro del GC, la profundidad de la cuenca es mayor que $3000 \mathrm{~m}$, por lo que hay un libre intercambio de agua con el océano Pacífico (Castro et al. 2000). En la parte sur de la RGI se encuentran el umbral de San Esteban ( $\sim 600 \mathrm{~m}$ de profundidad) y el umbral de San Lorenzo ( $400 \mathrm{~m}$ de profundidad) restringiendo la entrada de las masas de agua hacia el canal de Ballenas $(\sim 1500 \mathrm{~m}$ de profundidad). En el norte del canal de Ballenas se encuentra otro umbral que conecta con la cuenca Delfín ( $800 \mathrm{~m}$ de profundidad) (Badan-Dangon et al. 1991).

La campaña oceanográfica Umbrales I se realizó en la RGI a bordo del B/O Francisco de Ulloa en marzo de 2002, iniciando en mareas vivas y terminando en mareas muertas. La red de muestreo cubrió 38 estaciones (fig. 1a) donde se realizaron lances de CTD (Sea-Bird 911 Plus). En 26 de estas estaciones se recolectaron muestras de agua de mar con botellas Niskin en la superficie y a distintas profundidades $(5,10$, $20,30,50,75,100,200,250,300,400,500,700,750,800$, 900 y 1000 m), según lo permitiera la batimetría, para el análisis del CID. El agua de mar luego se estrajo de las botellas Niskin con jeringas de $50 \mathrm{~mL}$ y el CID se midió a bordo utilizando la técnica de titulación potenciométrica descrita por Hernández-Ayón et al. (1999).

Para analizar la variación temporal de la presencia de las masas de agua y su efecto sobre las concentraciones de CID, 
campaign in September 2004, which were coulometrically analyzed at Scripps Institution of Oceanography, University of California. The accuracy of the potentiometric and coulometric DIC measurements was \pm 10 and $\pm 3 \mu \mathrm{mol} \mathrm{kg}{ }^{-1}$, respectively

\section{Data processing}

Water masses were identified based on a temperaturesalinity (T-S) diagram and following the classification proposed by Torres-Orozco (1993). The stratification parameter $(\phi)$ was determined according to Simpson and Bowers (1981), in order to estimate the effect of vertical mixing on the surface distribution of DIC in the MIR. The value of $\phi\left(\mathrm{J} \mathrm{m}^{-3}\right)$ is a measure of the amount of energy required to mix a water column, high values indicating a more stratified water column and low values the opposite.

\section{RESULTS}

\section{Water masses in the Midriff Islands region}

The water masses detected in March 2002 in the MIR were GCW, SST, and PIW (fig. 2). GCW occupied all the surface layer of the MIR (fig. 2a), while SST occurred below $\sim 100 \mathrm{~m}$ in most of the region but not in the northwestern part se compararon los datos recolectados en marzo de 2002 (Umbrales I), mayo de 2003 (Umbrales II) y septiembre de 2004 (Umbrales III) a las mismas profundidades en tres estaciones representativas de la región del canal de Ballenas (fig 1a).

\section{Calibración de las concentraciones de CID durante la campaña Umbrales I}

Las concentraciones de CID medidas por potenciometría durante la campaña Umbrales I se calibraron con las mediciones de CID obtenidas de las muestras recolectadas en aguas profundas en la estación 38 de la campaña Umbrales II en mayo de 2003 y Umbrales III en septiembre de 2004, las cuales se analizaron mediante coulombimetría en Scripps Institution of Oceanography de la Universidad de California. La precisión de las mediciones de CID obtenidas por potenciometría fue de $\pm 10 \mu \mathrm{mol} \mathrm{kg}^{-1}$ y la de las mediciones obtenidas por coulombimetría fue de $\pm 3 \mu \mathrm{mol} \mathrm{kg}^{-1}$.

\section{Procesamiento de datos}

Las masas de agua se identificaron a partir de un diagrama de temperatura-salinidad (T-S) usando la clasificación de Torres-Orozco (1993). El parámetro de estratificación ( $\phi)$ se determinó de acuerdo con Simpson y Bowers (1981),
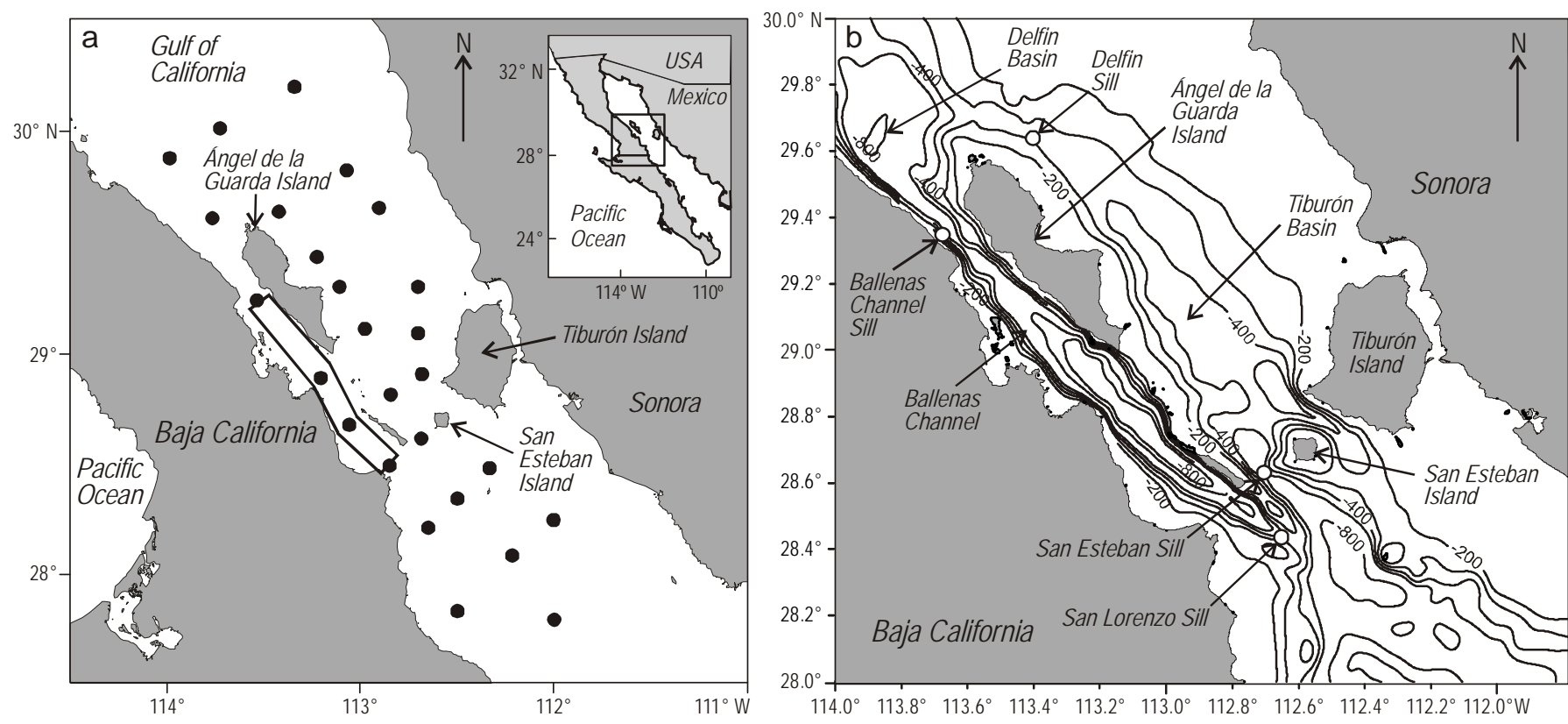

Figure 1. Map of the Midriff Islands region in the Gulf of California showing the grid of sampling stations of the Umbrales I campaign in March 2002. (a) Stations where CTD casts were made and dissolved inorganic carbon samples were collected (•); the rectangle indicates the stations considered in Ballenas Channel to compare between March 2002, May 2003, and September 2004. (b) Bathymetry of the region (taken from López and García 2003).

Figura 1. Mapa de la región de las grandes islas en el golfo de California donde se muestra la red de estaciones de muestreo de la campaña Umbrales I en marzo de 2002. (a) Estaciones donde se realizaron los lances de CTD y se recolectaron las muestras de carbono inorgánico disuelto (•); el rectángulo indica las estaciones en el canal de Ballenas que se compararon entre marzo de 2002, mayo de 2003 y septiembre de 2004. (b) Batimetría de la región (tomado de López y García 2003). 
off the coast of Sonora (fig. 2b). PIW occurred below $500 \mathrm{~m}$ depth and filled San Pedro Mártir Basin located in the vicinity of station 38, the southernmost station (fig. 1); unlike the other two water masses, PIW did not penetrate to the north of the sill (fig. 2c). Hence, in this survey, the chemical characteristics of the water below the euphotic zone likely depend on the proportions of the first two water masses and perhaps on a small proportion of PIW that may have become mixed with SSW due to the effect of the tide. The approximate proportions of the masses were $39 \%$ AGC, $44 \%$ SSW, and $16 \%$ PIW. A very small proportion, $1 \%$, of Pacific Deep Water (PDW) was also detected below PIW but only at the southernmost station and at $1200 \mathrm{~m}$ depth.

The characteristics presented by GCW were considered of particular interest. This water mass occupied the surface layer throughout the MIR, but its volume was generally greater to the northwest (on average in the first $170 \mathrm{~m}$ ) and decreased $\sim 85 \mathrm{~m}$ toward the southeast. This water mass appeared $V$-shaped in the T-S diagram (fig. 3), with maximum temperature values on the left arm of the $V$ and maximum salinity values on the right arm. The vertex was the coldest and less saline part, its stations coinciding with the upper limit of SSW. The T-S diagram showed three water types, which in March formed three oceanographic subregions associated with vertical mixing and residence times. These subregions were not only delimited at the surface (fig. 3b), but also vertically with different structures and grouped according to their temperature, salinity, and density (fig. 3e, f, g). The three water types were called GCW-1, 2, and 3 , indicating a gradient of greater to lesser mixing intensity or of lesser to greater stratification.

GCW-1 was found at the Ballenas Channel stations (the $V$ 's vertex), corresponding to the less stratified area, with $\phi$ values less than $17 \mathrm{~J} \mathrm{~m}^{-3}\left(\mathrm{~T}_{\text {mean }}=14.3 \pm 0.4^{\circ} \mathrm{C} ; \mathrm{S}_{\text {mean }}=\right.$ $35.05 \pm 0.03$ ) (fig. 3c, d). GCW-2 corresponded to the subregion with intermediate stratification, with $\phi$ values between 17 and $30 \mathrm{~J} \mathrm{~m}^{-3}\left(\mathrm{~T}_{\text {mean }}=16.0 \pm 0.3{ }^{\circ} \mathrm{C} ; \mathrm{S}_{\text {mean }}=35.31 \pm 0.07\right)$, to the northeast of the MIR and on the northwestern side of Ángel de la Guarda Island. GCW-3 corresponded to the most stratified area, with $\phi$ values between 30 and $\sim 75 \mathrm{~J} \mathrm{~m}^{-3}$ $\left(\mathrm{T}_{\text {mean }}=17.5 \pm 1.1^{\circ} \mathrm{C} ; \mathrm{S}_{\text {mean }}=35.20 \pm 0.04\right)$, to the southeast of the MIR. The $\phi v s$ surface temperature and salinity plots (fig. 3c, d) show that stratification was more closely related to temperature. The first case ( $\phi v s \mathrm{~T})$ may be an indication of mixing, but the second ( $\phi v s \mathrm{~S})$ is likely an indication of a response to the differences in residence times; that is, the longer water remains on the surface the saltier it becomes due to evaporation.

Below GCW, SSW showed two distinct distributions differentiated by temperature, salinity, and density (fig. 4a, b). In the northwestern part of the MIR, this water mass was detected below $200 \mathrm{~m}$, filling the Delfín, Salsipuedes, and Tiburón basins, while in the southeastern part it occurred between 150 and $350 \mathrm{~m}$ depth (fig. 2b). con la finalidad de estimar el efecto de la mezcla vertical sobre la distribución superficial de CID en la RGI. El valor de $\phi\left(\mathrm{J} \mathrm{m}^{-3}\right)$ representa una medida de la cantidad de energía que se requiere para mezclar una columna de agua; los valores altos indican una columna de agua más estratificada y los valores bajos indican lo contrario.

\section{Resultados}

\section{Masas de agua en la región de las grandes islas}

Las masas de agua que se detectaron en la RGI en marzo de 2002 fueron AGC, ASS y AIP (fig. 2). En general, se observó que el AGC cubrió toda la superficie de la RGI (fig. 2a), mientras que el ASS se localizó en una extensa porción por debajo de los $\sim 100 \mathrm{~m}$ pero sin cubrir la zona noroeste aledaña a la costa de Sonora (fig. 2b). El AIP se localizó por debajo de $500 \mathrm{~m}$ y llenó la cuenca de San Pedro Mártir localizada aproximadamente en la estación 38 (fig. 1). En contraste con las otras dos masas de agua, el AIP no atravesó hacia el norte del umbral (fig. 2c). Por lo tanto, en este muestreo se podría esperar que las características químicas del agua por debajo de la zona eufótica dependieran de las proporciones de las dos primeras masas de agua y quizás de una pequeña proporción del AIP que casualmente pudiera lograr mezclarse con el ASS por efecto de mareas. La proporción aproximada de las masas de agua fue de $39 \%$, $44 \%$ y $16 \%$ para AGC, ASS y AIP, respectivamente. También se detectó el Agua Profunda del Pacífico (APP) por debajo del AIP, pero su proporción representó únicamente el $1 \% \mathrm{y}$ sólo se observó en la estación más sureña y a $1200 \mathrm{~m}$ de profundidad.

El AGC presentó características que se consideran importantes describir. Esta masa de agua se localizó en toda la superficie de la RGI, pero su volumen fue mayor al noroeste (en los primeros $170 \mathrm{~m}$ en promedio) y disminuyó en $\sim 85 \mathrm{~m}$ hacia el sureste. Esta masa de agua presentó una forma de $V$ en el diagrama T-S (fig. 3), con valores máximos de temperatura en el brazo del lado izquierdo que conforma la $V$ y valores máximos de salinidad en el brazo derecho. El vértice fue la parte más fría y menos salina, cuyas estaciones coinciden con el límite superior de la ASS. El diagrama T-S mostró que el AGS estuvo formada por tres tipos de agua, las cuales durante marzo formaron tres subregiones oceanográficas asociadas al efecto de la mezcla vertical y los tiempos de residencia. Estas subregiones se delimitaron no sólo en la superficie (fig. 3b), sino también en la vertical con estructuras diferenciadas y agrupadas en función de su temperatura, salinidad y densidad (fig. 3e, f, g). En este trabajo, las tres subregiones se denominaron AGC-1, 2 y 3; esta clasificación también indica un gradiente de mayor a menor intensidad de la mezcla o de menor a mayor estratificación.

La AGC-1 se localizó en las estaciones correspondientes al canal de Ballenas (el vértice de la $V$ del diagrama T-S) y correspondió a la zona menos estratificada, con valores de $\phi$ 

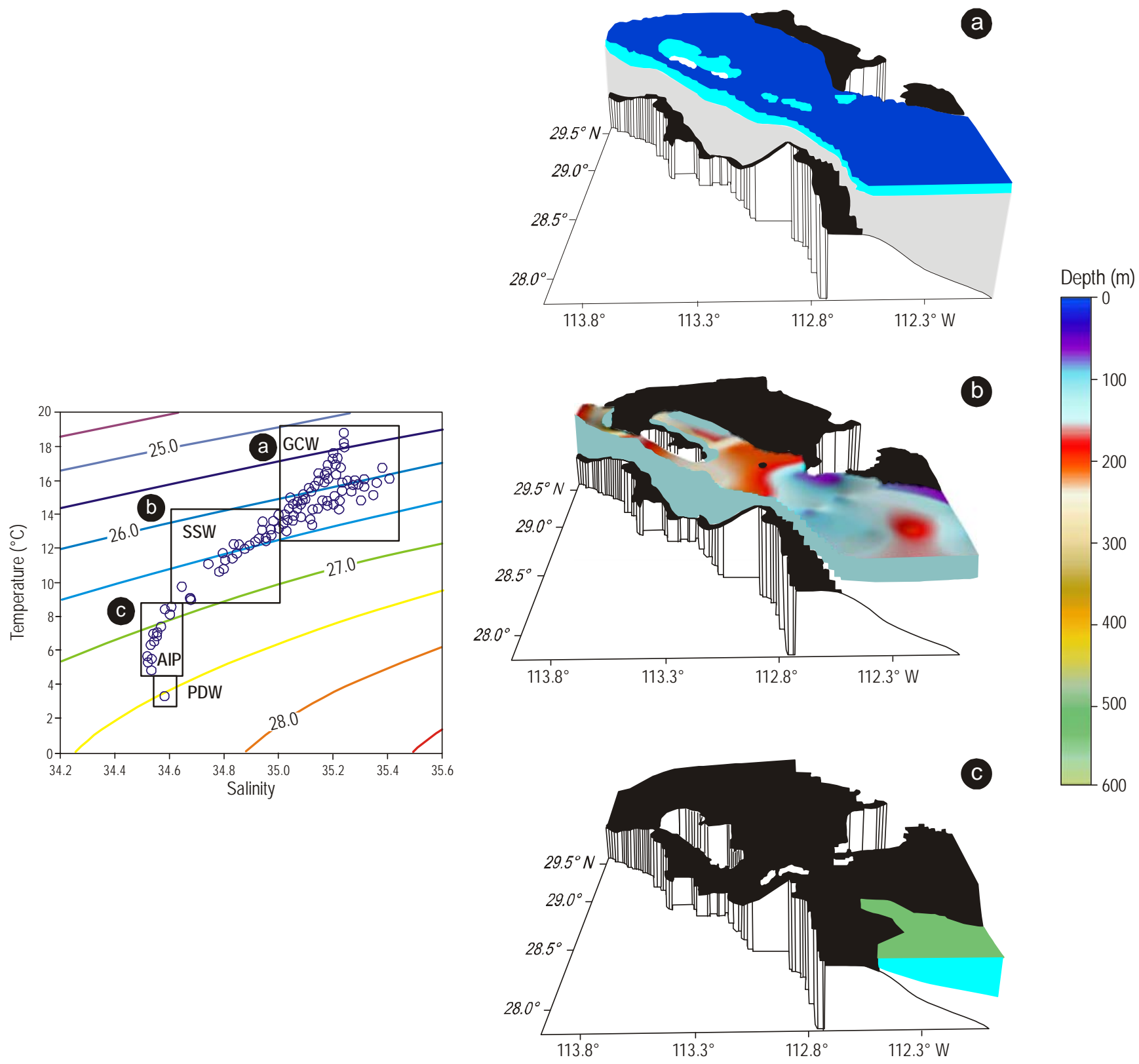

Figure 2. Water masses present in the Midriff Islands region and their spatial coverage. The T-S diagram shows the water masses identified in March 2002: Gulf of California Water (GCW), Subsurface Subtropical Water (SSW), and Pacific Intermediate Water (PIW). The letters $a, b$, and $c$ in the T-S diagram relate to the breadth and depth representations for each water mass on the right. The estimated proportions of the water masses were $39 \%, 44 \%$, and $16 \%$ for GCW, SSW, and AIP, respectively. Pacific Deep Water (PDW, $1 \%$ of the volume) was detected to the south of the region.

Figura 2. Masas de agua presentes en la región de las grandes islas y su cobertura espacial. El diagrama T-S muestra las masas de agua identificadas en marzo de 2002: Agua del Golfo de California (GCW), Agua Subsuperficial Subtropical (SSW) y Agua Intermedia del Pacífico (PIW). Las letras $a, b$ y $c$ en el diagrama T-S se relacionan con las representaciones de anchura y profundidad para cada masa de agua del lado derecho. La proporción estimada para cada masa de agua fue 39\%, 44\% y $16 \%$ de GCW, SSW y AIP, respectivamente. Se detectó Agua Profunda del Pacífico (PDW, 1\% del volumen) hacia el sur de la región. 

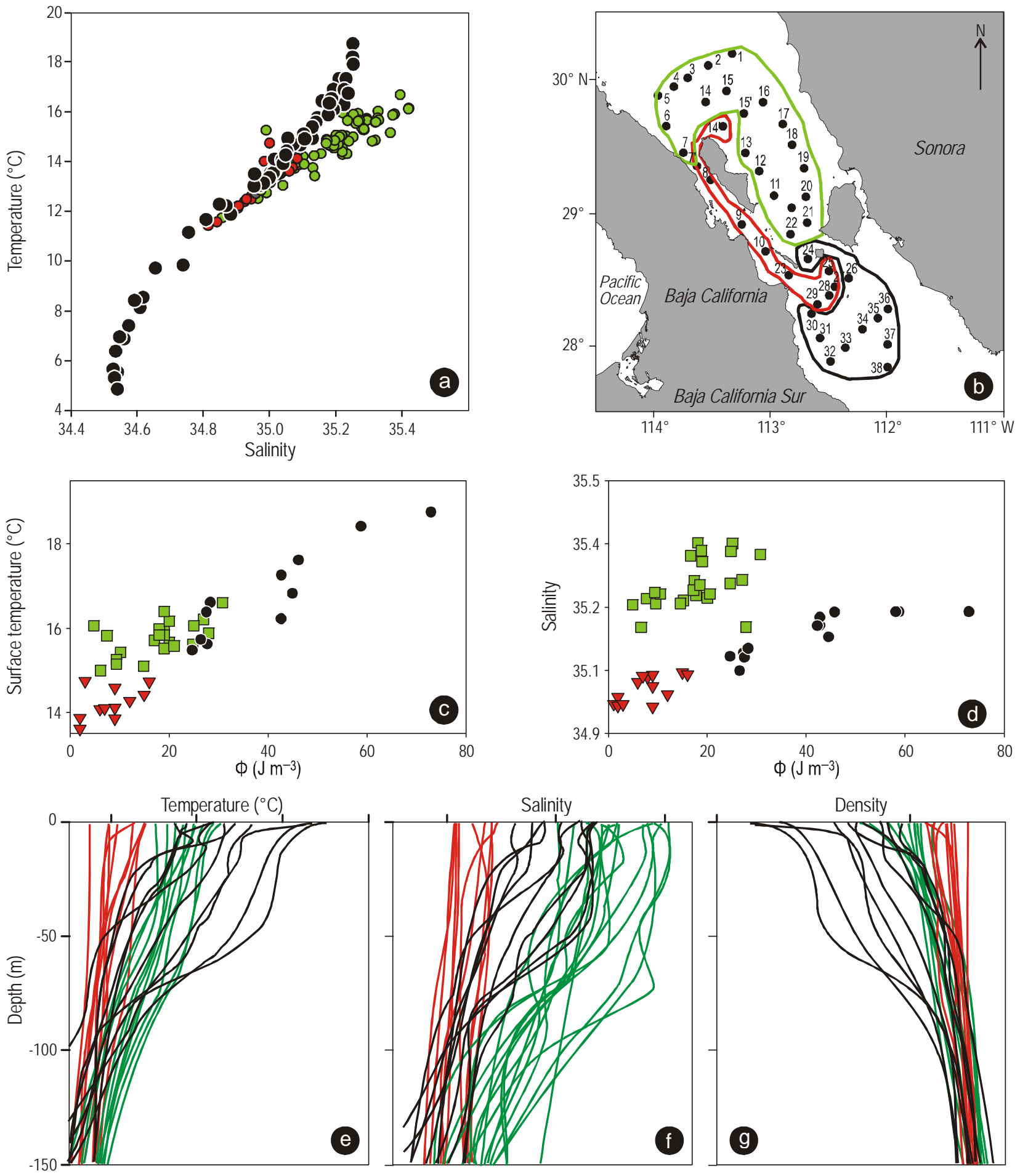

Figure 3. (a) T-S diagram for the Umbrales I campaign. (b) Three subregions identified within Gulf of California Water (GCW) based on their temperature and salinity properties: GCW-1 (red), GCW-2 (green), and GCW-3 (black). (c) Temperature vs stratification parameter ( $\phi$ ) and (d) salinity $v s \phi$ relationship. (e) Potential temperature, (f) salinity, and (g) density.

Figura 3. (a) Diagrama T-S para la campaña Umbrales I. (b) Las tres subregiones identificadas a partir de su temperatura y salinidad en la capa del Agua del Golfo de California (GCW): GCW-1 (rojo), GCW-2 (verde) y GCW-3 (negro). (c) Relación temperatura vs parámetro de estratificación $(\phi)$ y (d) salinidad vs $\phi$. (e) Temperatura potencial, (f) salinidad y (g) densidad. 

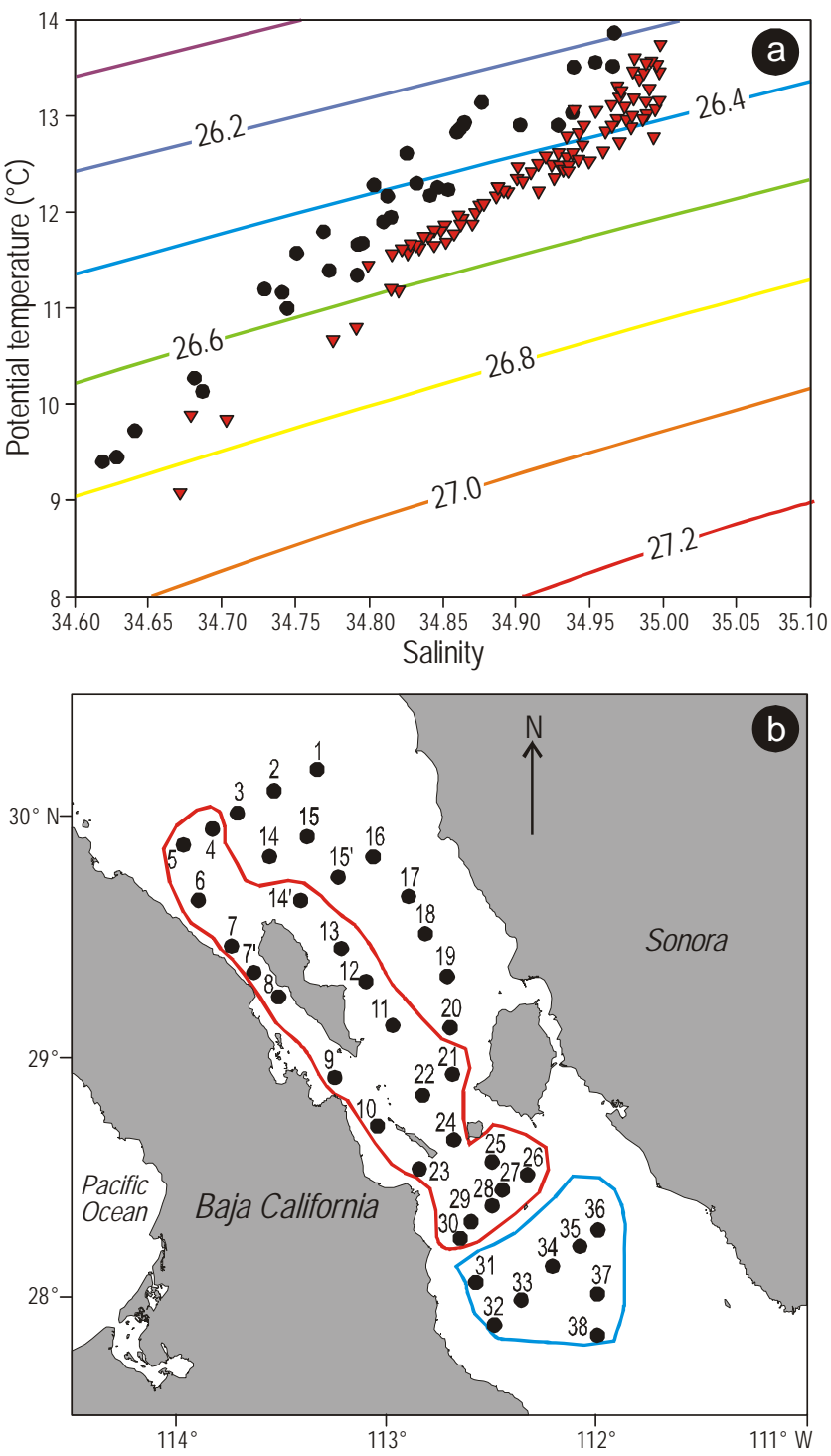

Figure 4. (a) T-S diagram for Subtropical Subsurface Water (SSW) in the Midriff Islands region. A group of data is separated by temperature and salinity properties: higher $(\boldsymbol{\nabla})$ and lower $(\bullet)$ density (color lines). (b) Map showing the spatial distribution of SSW inside (higher density, red line) and outside (lower density, blue line) the region.

Figura 4. (a) Diagrama T-S para el Agua Subsuperfial Subtropical (ASS) en la región de las grandes islas. Se muestra un grupo de datos separados por propiedades de temperatura y salinidad: mayor ( $\boldsymbol{\nabla})$ y menor $(\bullet)$ densidad (líneas de color). (b) Mapa donde se muestra la distribución espacial del ASS dentro (mayor densidad, línea roja) y fuera (menor densidad, línea azul) de la región.

\section{DIC and hydrographic characteristics}

Each water mass in the MIR presented different DIC, temperature, salinity, and density properties (fig. 5). Of the three water masses, GCW had the lowest concentrations of DIC, ranging from 2250 to $2150 \mu \mathrm{mol} \mathrm{kg}^{-1}$ (fig. 5a). menores que $17 \mathrm{~J} \mathrm{~m}^{-3}\left(\mathrm{~T}_{\text {media }}=14.3 \pm 0.4{ }^{\circ} \mathrm{C} ; \mathrm{S}_{\text {media }}=35.05 \pm\right.$ 0.03 ) (fig. 3c, d). La AGC-2 correspondió a la subregión con estratificación intermedia, con valores de $\phi$ entre 17 y $30 \mathrm{~J}$ $\mathrm{m}^{-3}\left(\mathrm{~T}_{\text {media }}=16.0 \pm 0.3^{\circ} \mathrm{C} ; \mathrm{S}_{\text {media }}=35.31 \pm 0.07\right)$ al noroeste de la RGI y en el lado nororiental de la isla Ángel de la Guarda. La AGC-3 correspondió a la zona más estratificada, con valores de $\phi$ entre $30 \mathrm{y} \sim 75 \mathrm{~J} \mathrm{~m}^{-3}\left(\mathrm{~T}_{\text {media }}=17.5 \pm 1.1{ }^{\circ} \mathrm{C}\right.$; $\left.\mathrm{S}_{\text {media }}=35.20 \pm 0.04\right)$ al sureste de la RGI. Al graficar $\phi$ contra la temperatura y la salinidad superficial (fig. $3 \mathrm{c}, \mathrm{d}$ ), se observó que la estratificación estuvo más asociada con la temperatura. El primer caso ( $\phi v s \mathrm{~T})$ pudiera ser una indicación de mezcla, pero el segundo ( $\phi$ vs $\mathrm{S}$ ) posiblemente sea una indicación de una respuesta a las diferencias en el tiempo de residencia, es decir, que el agua tenga un mayor tiempo de exposición en la superficie y que su salinidad haya aumentado por la evaporación.

Por debajo del AGC, el ASS presentó dos distribuciones distintas visiblemente diferenciadas por temperatura, salinidad y densidad (fig. 4a, b). En el noroeste de la RGI, esta masa de agua se observó por debajo de los $200 \mathrm{~m}$, llenando las cuencas Delfín, Salsipuedes y Tiburón, y en el sureste se localizó entre 150 y $350 \mathrm{~m}$ de profundidad (fig. 2b).

\section{Características hidrográficas y del CID}

Cada masa de agua en la RGI presentó características distintas de temperatura, salinidad, densidad y CID (fig. 5). El AGC presentó las menores concentraciones de CID (de 2250 a $2150 \mu \mathrm{mol} \mathrm{kg}-1$ ), en comparación con las otras dos masas de agua (fig. 5a). Se observó una distribución de CID de acuerdo con las tres subregiones oceanográficas: en la AGC-1 fueron mayores $\left(\sim 2300 \mu \mathrm{mol} \mathrm{kg}{ }^{-1}\right)$, en la AGC-2 fluctuaron entre $2300 \mathrm{y} \sim 2250 \mu \mathrm{mol} \mathrm{kg}{ }^{-1}$, y en la AGC-3

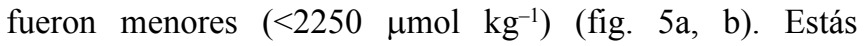
subregiones oceanográficas también fueron clasificadas de acuerdo con la forma de la estructura vertical de las diferentes variables (separación de las más mezcladas y las más estratificadas) (fig. 6). Los perfiles de temperatura, salinidad, densidad y CID en los primeros $1000 \mathrm{~m}$ permitieron evaluar diversos aspectos. (1) En la AGC-1 (canal de Ballenas), la columna de agua estuvo muy bien mezclada, al grado de que las diferencias en las variables, entre la superficie y los $200 \mathrm{~m}$ de profundidad, fueron pequeñas $\left(2{ }^{\circ} \mathrm{C}, 0.2\right.$ de salinidad, $0.5 \mathrm{~kg} \mathrm{~m}^{-3}$ de densidad y $<30 \mu$ mol $\mathrm{kg}^{-1}$ de CID). Además, los valores de referencia del diagrama T-S $\left(14^{\circ} \mathrm{C}\right.$ y S $\left.<35\right)$ indicaron que, en esta subregión, el ASS se presentó en toda la columna de agua. Por lo tanto, las altas concentraciones de CID son producto de la intensidad de mezcla, pero están influenciadas por la presencia somera del ASS, que de origen es rica en CID. (2) En la AGC-3 (localizada al sur de la RGI) se observó un aumento en la estratificación como resultado de la disminución del efecto de mezcla, y las concentraciones de CID en la superficie fueron menores; sin embargo, por debajo de los $\sim 400 \mathrm{~m}$ se observó la presencia del AIP, que es más fría, menos salina y aún más rica en CID. 
Regarding the three oceanographic subregions, DIC concentrations were highest in GCW-1 $\left(\sim 2300 \mu \mathrm{mol} \mathrm{kg}^{-1}\right)$, ranged from 2300 to $\sim 2250 \mu \mathrm{mol} \mathrm{kg}^{-1}$ in GCW-2, and were lowest in GCW-3 $\left(<2250 \mu \mathrm{mol} \mathrm{kg}{ }^{-1}\right)$ (fig. 5a, b). These subregions were also classified in relation to the vertical structure of the different variables (separating the most mixed and most stratified) (fig. 6). The 1000-m temperature, salinity, density, and DIC profiles allowed the following assessment. (1) In GCW-1 (Ballenas Channel), the water column was well mixed and there was little difference in the variables between the surface and $200 \mathrm{~m}$ depth $\left(2^{\circ} \mathrm{C}, 0.2\right.$ salinity,

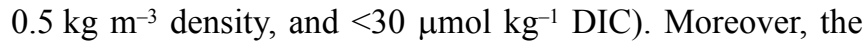
reference values of the T-S diagram $\left(14{ }^{\circ} \mathrm{C}, \mathrm{S}<35\right)$ indicated that, in this subregion, SSW was present throughout the water column. Therefore, the high DIC concentrations were due to the intensity of mixing, but influenced by the shallow presence of DIC-rich SSW. (2) In GCW-3 (southern part of the MIR), there was an increase in stratification due to a reduction in mixing and surface DIC concentrations were lower; however, below $\sim 400 \mathrm{~m}$, PIW was observed, which is colder, more saline, and even richer in DIC.

\section{Temporal variation of DIC in Ballenas Channel}

In Ballenas Channel, SSW occurred in close proximity to the surface waters $(\sim 50 \mathrm{~m})$ in March (late winter); however, this scenario varied in other seasons (fig. 7).

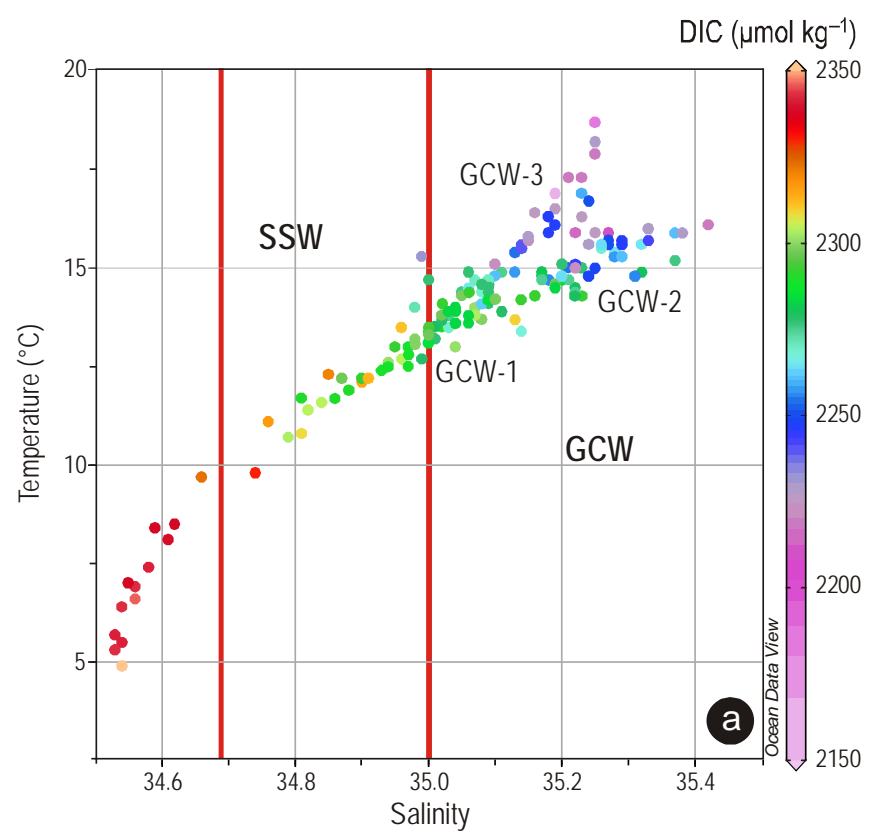

\section{Variación temporal del CID en el canal de Ballenas}

En el canal de Ballenas el ASS en marzo (finales de invierno) se observó muy cerca de las aguas superficiales ( $\sim 50 \mathrm{~m})$; sin embargo, este escenario varió en las otras épocas del año (fig. 7).

En marzo (invierno), la temperatura superficial fue de $\sim 14{ }^{\circ} \mathrm{C}$ y las salinidades estuvieron ligeramente por arriba de 35 ; además, se presentó una columna de agua bien mezclada. En mayo (primavera), la temperatura y la salinidad aumentaron $\sim 6^{\circ} \mathrm{C}$ y $\sim 0.4$, respectivamente, en las aguas más superficiales, mientras que la densidad disminuyó una unidad y

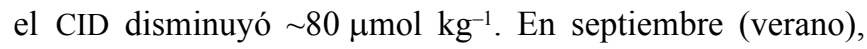
las diferencias fueron más evidentes en las aguas superficiales (ver amplificación para cada variable en fig. 7): la temperatura aumentó más de $14{ }^{\circ} \mathrm{C}$, la salinidad aumentó en más de una unidad y, por lo tanto, la densidad disminuyó más de $4 \mathrm{~kg} \mathrm{~m}^{-3}$, mientras que el CID disminuyó en $\sim 200 \mu \mathrm{mol} \mathrm{kg}{ }^{-1}$. Es importante señalar que las diferencias estacionales se observaron principalmente en los primeros $200 \mathrm{~m}$ de profundidad, pero las diferencias tendieron a desaparecer de manera gradual hacia el fondo (fig. 7).

Hacia el verano, además de presentarse mayor estratificación, se observó mayor presencia del AGC en los primeros $100 \mathrm{~m}$ (con base en los valores de T-S), donde dicha masa de agua, al ser menos densa, contribuyó con el hundimiento gradual del ASS. En marzo, el ASS se observó cerca de los

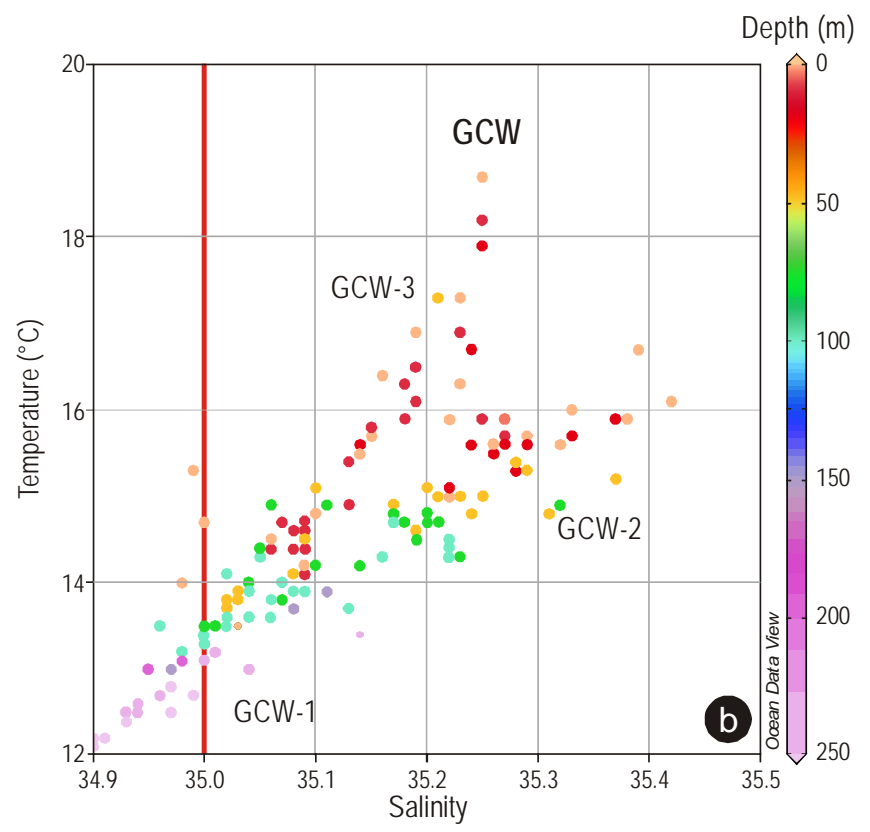

Figure 5. (a) T-S diagram. Color code for dissolved inorganic carbon (DIC) concentrations of Gulf of California Water (GCW) and Subtropical Subsurface Water (SSW). Subregions (based on their salinity and temperature properties) within GCW are indicated. (b) Blow-up of the T-S diagram for the GCW region. Color code for depth. GCW-3 represents a depth of $50 \mathrm{~m}$.

Figura 5. (a) Diagrama T-S. Código de color para las concentraciones de carbono inorgánico disuelto (DIC) del Agua del Golfo de California Water (GCW) y Agua Subtropical Subsuperficial (SSW). Se muestran las subregiones (según sus propiedades de salinidad y temperatura) dentro del GCW. (b) Ampliación del diagrama T-S para la región del GCW. Código de color para la profundidad. GCW-3 representa los primeros $50 \mathrm{~m}$ de profundidad. 

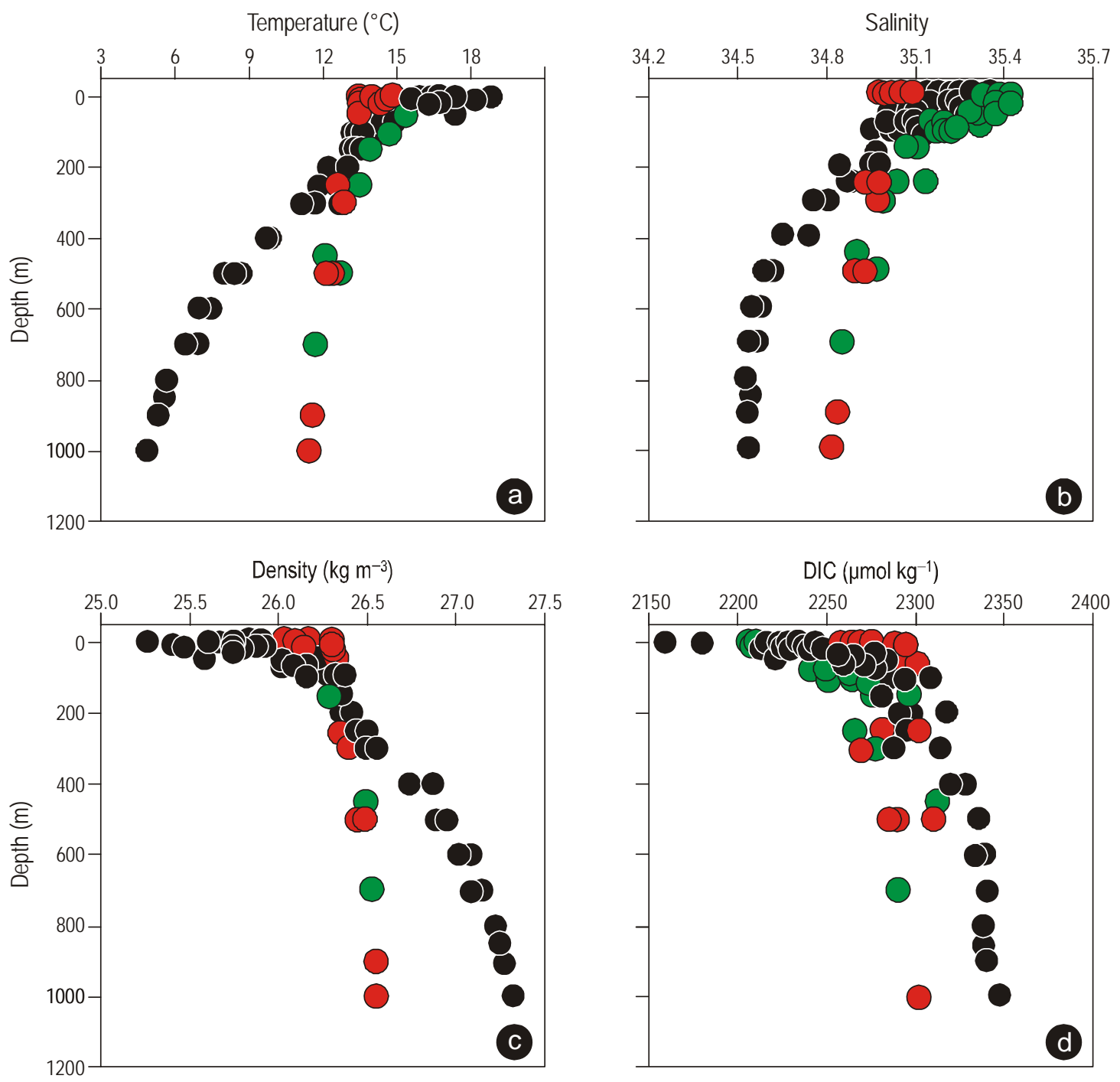

Figure 6. Vertical structure of (a) temperature, (b) salinity, (c) density, and (d) dissolved inorganic carbon (DIC) in the Midriff Islands region; GCW-1 in red, GCW-2 in green, and GCW-3 in black. Water below 400 m corresponds to Pacific Intermediate Water, with temperature < $9^{\circ} \mathrm{C}$, density $>26$, and DIC $>2330 \mu \mathrm{mol} \mathrm{kg}{ }^{-1}$. Above $400 \mathrm{~m}$, Subtropical Subsurface Water predominates.

Figura 6. Estructura vertical de (a) temperatura, (b) salinidad, (c) densidad y (d) carbono inorgánico disuelto (DIC) en la región de las grandes islas; GCW-1 en rojo, GCW-2 en verde y GCW-3 en negro. El agua por debajo de los $400 \mathrm{~m}$ corresponde al Agua Intermedia del Pacífico, con temperatura $<9{ }^{\circ} \mathrm{C}$, densidad $>26$ y DIC $>2330 \mu \mathrm{mol} \mathrm{kg} \mathrm{kg}^{-1}$. Por arriba de los $400 \mathrm{~m}$ predomina el Agua Subtropical Subsuperficial.

In March (winter), sea surface temperature was $\sim 14^{\circ} \mathrm{C}$, salinity was slightly higher than 35 , and the water column was well mixed. In May (spring), temperature and salinity increased $\sim 6{ }^{\circ} \mathrm{C}$ and $\sim 0.4$, respectively, in the waters closest to the surface, while density decreased by one unit and DIC decreased by $\sim 80 \mu \mathrm{mol} \mathrm{kg}{ }^{-1}$. In September (summer), the differences were more evident in surface waters (see amplification for each variable in fig. 7); temperature increased more than $14{ }^{\circ} \mathrm{C}$, salinity increased by more than one unit and, therefore, density decreased more than $4 \mathrm{~kg} \mathrm{~m}^{-3}$, while DIC decreased by $\sim 200 \mu \mathrm{mol} \mathrm{kg}{ }^{-1}$. Note that the seasonal differences were more noticeable in the upper $200 \mathrm{~m}$ and tended to disappear gradually towards the bottom (fig. 7).
$70 \mathrm{~m}$, en mayo se observó por debajo de los $\sim 100 \mathrm{~m}$ y en septiembre se hundió hasta los $200 \mathrm{~m}$ de profundidad. Se observó que al disminuir la influencia del ASS, también disminuyó la concentración de CID en la superficie (fig. 7c, d).

\section{DISCUSIÓN}

La RGI presenta las temperaturas superficiales más bajas del GC debido a la intensa mezcla por las mareas (Argote et al. 1995, Paden et al. 1993, Soto-Mardones et al. 1999). Esta mezcla favorece el transporte de aguas profundas, con bajas temperaturas y altas concentraciones de nutrientes y metales hacia la superficie (Delgadillo-Hinojosa et al. 2001, 
Towards summertime, in addition to increased stratification, there was greater presence of GCW in the first $100 \mathrm{~m}$ (based on the T-S values) and this less dense water mass contributed to the gradual sinking of SSW. SSW was observed at $70 \mathrm{~m}$ depth in March and below $\sim 100 \mathrm{~m}$ in May, sinking to $200 \mathrm{~m}$ depth in September. As the influence of SSW diminished, so did the surface DIC concentration (fig. 7c, d).

\section{Discussion}

The MIR has the coldest surface temperatures of the GC because of intense tidal mixing (Argote et al. 1995, Paden et al. 1993, Soto-Mardones et al. 1999), which transports cold, deep, nutrient- and metal-rich waters to the surface (Delgadillo-Hinojosa et al. 2001, Torres-Valdés 2000). High
Torres-Valdés 2000). También se han registrado niveles altos de $\mathrm{pCO}_{2}$ en toda la RGI, por lo que la región se considera una fuente de $\mathrm{CO}_{2}$ hacia la atmósfera (Hidalgo-González et al. 1997, Zirino et al. 1997, Hernández-Ayón 2007b). Sin embargo, la mayoría de los trabajos previos sólo hacen alusión al mecanismo de mezcla como regulador de la composición química de las aguas superficiales de la RGI, sin considerar el papel que pudieran jugar las masas de agua, las cuales presentan una clara dinámica estacional.

En este trabajo, se detectaron dos masas de agua principales: el AGC, con una propoporción de 39\% del volumen total estimado, y el ASS, con una propoporción del 44\%. La composición de CID, salinidad, temperatura (fig. 5) y oxígeno disuelto (datos no presentados) de cada una de estas masas de agua fue muy distinta; por ejemplo, en invierno, el CID
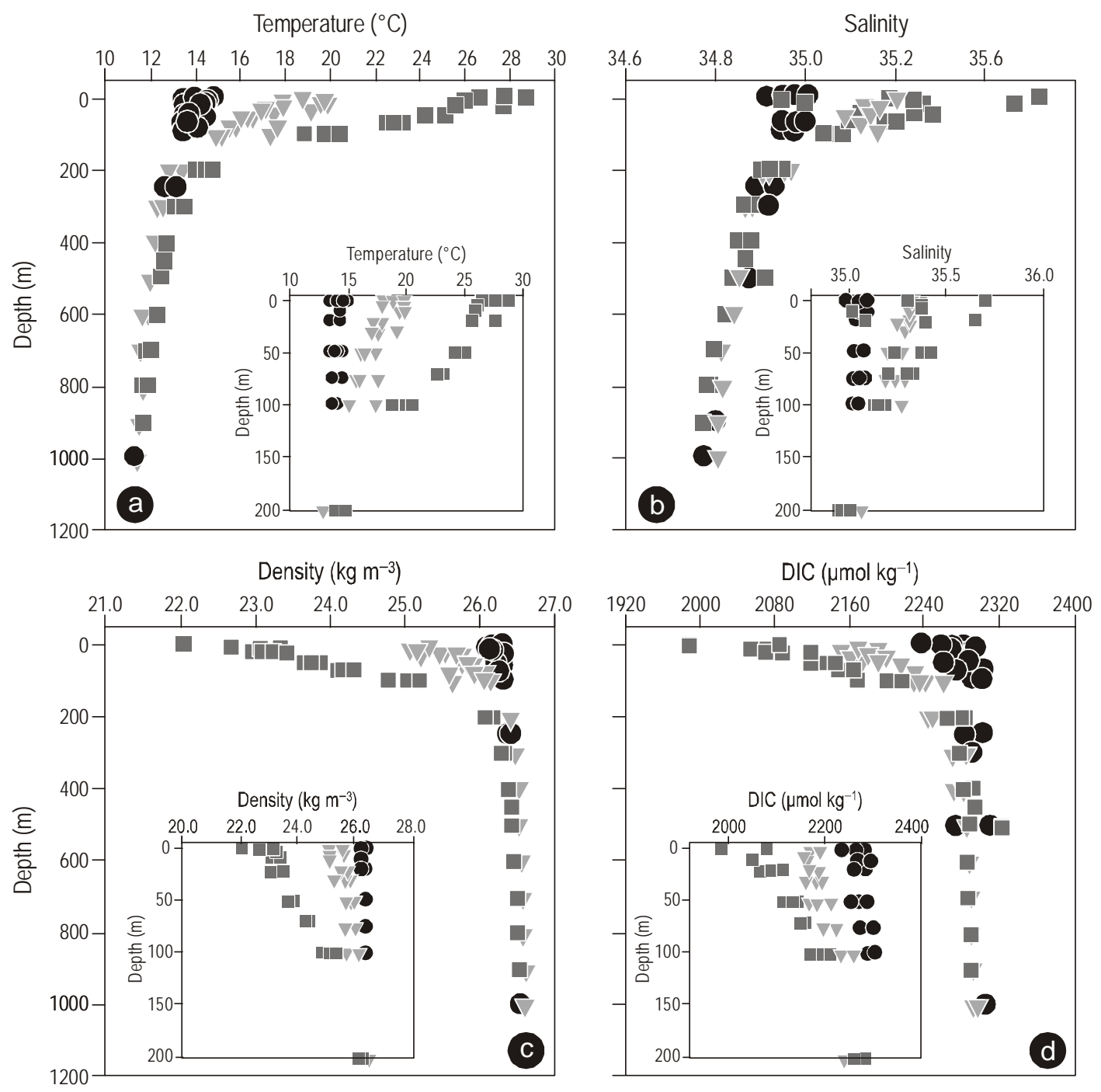

Figure 7. Comparison of the (a) temperature, (b) salinity, (c) density, and (d) dissolved inorganic carbon (DIC) profiles for Ballenas Channel in March $2002(\bullet)$, April $2003(\boldsymbol{\nabla})$, and September 2004 (ם). The inset in each panel is a 200-m profile.

Figura 7. Comparación de los perfiles de (a) temperatura, (b) salinidad, (c) densidad y (d) carbono inorgánico disuelto (DIC) para el canal de Ballenas en marzo de $2002(\bullet)$, abril de 2003 ( $\boldsymbol{\nabla}$ ) y septiembre de 2004 ( $\mathbf{\square})$. El inserto en cada gráfica es un perfil de $200 \mathrm{~m}$. 
levels of $\mathrm{pCO}_{2}$ have also been recorded in the MIR and it is considered a source of atmospheric $\mathrm{CO}_{2}$ (Hidalgo-González et al. 1997, Zirino et al. 1997, Hernández-Ayón 2007b). Most previous studies, however, mention that the chemical composition of surface waters in the MIR is regulated by vertical mixing and do not consider the possible role of water masses, which present clear seasonal dynamics.

In the present study, two main water masses were detected: GCW, with an estimated proportion of $39 \%$ of the total estimated volume, and SSW, with a proportion of $44 \%$. The composition of DIC, salinity, temperature (fig. 5), and dissolved oxygen (data not presented) of these water masses differed; for example, in winter, DIC ranged from 1980 to $2160 \mu \mathrm{mol} \mathrm{kg}^{-1}$ in GCW but was $>2300 \mu \mathrm{mol} \mathrm{kg}^{-1}$ in SSW. There was evidence of the influence of SSW on the spatial distribution of DIC concentrations. The clearest signal was detected in surface waters of Ballenas Channel (subregion GCW-1), where DIC concentrations were maximum. A weaker signal was observed in the rest of the MIR (subregions GCW-2 and 3, fig. 5) and will be explained further on.

It was also possible to establish that the proportion of SSW in Ballenas Channel showed seasonal vertical variation. In winter SSW was detected close to the surface at $\sim 70 \mathrm{~m}$ (enriching all the water column with DIC, by $\sim 2300 \mu \mathrm{mol}$ $\mathrm{kg}^{-1}$ ), in summer it was detected below $\sim 200 \mathrm{~m}$, and in spring it was found at more than $100 \mathrm{~m}$ depth. This information can be used to explain several oceanographic aspects related to "enrichment."

As mixing in the MIR is constant year-round because it is tide-related (Flores-de-Santiago et al. 2007) and satellite images have revealed that in winter the coldest temperatures occur in Ballenas Channel (Santamaría-del-Angel et al. 1994, Flores-de-Santiago et al. 2007), the nutrient and DIC content of surface water will thus depend on the mixing ratio of SSW and surface water. For example, DIC concentrations in surface waters of the MIR will be higher when SSW is closer to the surface.

In the MIR, the effects of tidal mixing can be observed in the first $200 \mathrm{~m}$, but in Ballenas Channel, in March, the effects seem to extend to below $500 \mathrm{~m}$ depth. Our findings indicate the occurrence of a combination of factors in winter. SSW occurs in the first $100 \mathrm{~m}$ and as it is in reach of the effects of tidal mixing in the upper $200 \mathrm{~m}$, the water column loses stratification; this well-mixed water volume mixes with deeper (below $200 \mathrm{~m}$ ) unstratified water and generates a well-mixed water column from the surface to $>500 \mathrm{~m}$. López et al. (2006) and Marinone (2007) reported that in addition to tidal mixing, upwelling occurs in Ballenas Channel, bringing cold deep waters to the surface. This upwelling is generated by the convergence of deep water entering the channel from the south through the San Lorenzo sill and from the north through the Ballenas Channel sill. As a result of the very strong mixing generated in the MIR, the physical and chemical variables are highly correlated. The significant correlation $(P \leq 0.001)$ found between DIC and temperature $\left(r^{2}>0.9\right.$, fluctuó entre 1980 y $2160 \mu \mathrm{mol} \mathrm{\textrm {kg } ^ { - 1 }}$ en el AGC pero fue

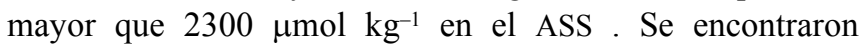
evidencias de la influencia de ASS en la distribución espacial de las concentraciones de CID. La señal más clara se detectó en las aguas superficiales del canal de Ballenas (subregión AGC-1), donde se registraron las concentraciones máximas de CID. En el resto de la RGI (subregiones AGC-2 y 3, fig. 5), se detectó una influencia más débil que será explicada más delante.

Se pudo establecer también que la proporción del ASS en el canal de Ballenas varía estacionalmente en la vertical. Mientras que en invierno el ASS se observó cerca de la superficie a $\sim 70 \mathrm{~m}$ de profundidad (enriqueciendo con CID, en $\sim 2300 \mu \mathrm{mol} \mathrm{kg}{ }^{-1}$, a toda la columna de agua), en verano su presencia se detectó por debajo de los $\sim 200 \mathrm{~m}$. En primavera el ASS se observó a más de $100 \mathrm{~m}$ de profundidad. Con la información obtenida, se pueden explicar varios aspectos oceanográficos relacionados con el tema de "enriquecimiento".

La mezcla en la RGI es constante a lo largo del año, ya que está relacionado con el efecto de mareas (Flores-deSantiago et al. 2007). En particular, en invierno se han detectado, vía imágenes de satélite, las temperaturas más bajas en el canal de Ballenas (Santamaría-del-Angel et al. 1994, Flores-de-Santiago et al. 2007). Por lo tanto, la composición del agua superficial, en términos de nutrientes y CID, dependerá de las proporciones en que el ASS se mezcle con el agua superficial; por ejemplo, habrá más CID en las aguas superficiales de la RGI cuando la masa de ASS se acerque a la superficie.

En la RGI, el efecto de la mezcla por mareas se puede apreciar en los primeros $200 \mathrm{~m}$ de profundidad, pero en el canal de Ballenas, en marzo, el efecto de la mezcla pareciera estar actuando hasta profundidades mayores que $500 \mathrm{~m}$. Sin embargo, con base en lo observado en este trabajo, creemos que en invierno ocurre una combinación de factores: el ASS se ubica en los primeros $100 \mathrm{~m}$ y al estar al alcance del efecto de la mezcla por mareas en los primeros $200 \mathrm{~m}$, se pierde la estratificación en la columna de agua; este volumen de agua bien mezclado se une con las aguas profundas (por debajo de $200 \mathrm{~m}$ ), en las cuales no hay estratificación, y genera una columna de agua muy bien mezclada desde la superficie hasta más de $500 \mathrm{~m}$ de profundidad. López et al. (2006) y Marinone (2007) documentaron que además de la mezcla por mareas, existe una surgencia en el canal de Ballenas que lleva agua profunda a la superficie. Esta surgencia se genera por la convergencia del agua profunda que entra desde el sur por el umbral de San Lorenzo y desde el norte por el umbral canal de Ballenas. La mezcla es tan fuerte en la RGI que existe una alta correlación entre las variables físicas y químicas. La correlación significativa $(P \leq 0.001)$ encontrada entre el CID y la temperatura $\left(r^{2}>0.9, n=562\right)$ se puede explicar por el transporte de aguas profundas de baja temperatura (Lopez et al. 2006, Marinone 2007) y ricas en CID, las cuales se 
$n=562)$ can be explained by the transport of deep, cold (López et al. 2006, Marinone 2007), DIC-rich waters, which become warmer as they rise to the surface. We will address this point again further on.

\section{Influence of SSW on the spatial distribution of DIC and GCW in winter}

The wide sampling area allowed a detailed analysis of the evolution and influence of GCW on the oceanographic conditions of the MIR. The surface data indicate high DIC concentrations throughout the MIR, ranging from 2200 to $2300 \mu \mathrm{mol} \mathrm{kg} \mathrm{kg}^{-1}$. These concentrations are higher than in areas were advection of subsurface waters occurs. Off the west coast of Baja California, upwelled waters tend to have DIC concentrations of $\sim 2150 \mu \mathrm{mol} \mathrm{kg}{ }^{-1}$ and maximum $\mathrm{pCO}_{2}$ values of $800 \mu \mathrm{atm}$ (Ribas-Ribas et al. 2011), considering that the water typically comes from $\sim 80 \mathrm{~m}$ (Linacre et al. 2010). Values of $\sim 2200 \mu \mathrm{mol} \mathrm{kg}{ }^{-1}$ and $\mathrm{pCO}_{2}>900 \mu \mathrm{atm}$ have been reported for upwelled water off Oregon, where the winds are more intense $\left(10 \mathrm{~m} \mathrm{~s}^{-1}\right)$ and the water is transported from $\sim 200$ m (Feely et al. 2008). Hence, our DIC values for the MIR indicate that this area behaves as a source of $\mathrm{CO}_{2}$ to the atmosphere. It has been estimated that $\mathrm{pCO}_{2}$ values in Ballenas Channel can be as high as $\sim 1000 \mu$ atm (HernándezAyón et al. 2007b). Similar high values have been recorded off the coast of Peru during the upwelling season (Friederich et al. 2008).

DIC enrichment has mainly been attributed to factors such as little or no stratification (low $\phi$ values) caused by strong tidal currents (López and García 2003; TorresDelgado et al. 2013, this issue), as well as to tidal conditions, with direct effects on $\mathrm{CO}_{2}$ fluxes towards the atmosphere in the MIR (Hidalgo-González et al. 1997). For example, minimum stratification occurs two to three days after spring tides (Simpson and Bowers 1981). In addition to tidal mixing, particularly DIC (and nutrient) enrichment of surface waters in Ballenas Channel can also be explained by upwelling of deep water due to the convergence of SSW inflows from the south through San Lorenzo sill and from the north through Ballenas Channel sill (López et al. 2006, Marinone 2007). Marinone (2007) suggested that this upwelling is intense and occurs all year round.

Our findings show the role of SSW as a source of DIC to GCW when it increases in volume and tidal mixing occurs. At the surface, however, DIC concentration will also depend on the biogeochemical processes occurring there. GCW showed three water types forming three different hydrographic subregions, with particular temperature, salinity, and DIC characteristics that were, to a greater or lesser extent, related to SSW and other processes as discussed below.

GCW-1, located to the south of the islands and in Ballenas Channel, had the highest concentration of DIC. The most intense tidal currents occur in this area (MarinoneMoschetto and Lavín-Peregrina 2003), where the water is van calentando conforme llegan a la superficie. Este punto se retomará nuevamente más adelante.

\section{Influencia invernal del ASS en la distribución espacial del CID del AGC}

En este estudio la amplia malla de muestreo permitió describir de manera detallada la evolución e influencia del AGC sobre las condiciones oceanográficas de la RGI. Los datos superficiales muestran que en toda la RGI se encontraron concentraciones altas de CID que fluctuaron entre los $2200 \mathrm{y}$ $2300 \mu \mathrm{mol} \mathrm{\textrm {kg } ^ { - 1 }}$. Estas concentraciones son más altas que en zonas donde existe advección de aguas subsuperficiales, tal como es el caso de las aguas de surgencia de las costas occidentales de Baja California, cuyas concentraciones de CID suelen ser de $\sim 2150 \mu \mathrm{mol} \mathrm{kg}^{-1}$ y cuyos valores máximos de $\mathrm{pCO}_{2}$ son de $800 \mu$ atm (Ribas-Ribas et al. 2011), considerando que el agua proviene típicamente de $\sim 80 \mathrm{~m}$ (Linacre $e t$ al. 2010). En las aguas de surgencia de Oregón, donde los vientos son de mayor intensidad $\left(10 \mathrm{~m} \mathrm{~s}^{-1}\right)$ y el agua proviene de $\sim 200 \mathrm{~m}$, se han registrado concentraciones de CID de hasta $\sim 2200 \mu \mathrm{mol} \mathrm{kg}{ }^{-1}$ y valores de $\mathrm{pCO}_{2}>900 \mu$ atm (Feely et al. 2008). Por lo tanto, los valores de CID que encontramos para la RGI permiten entender que este sitio se comporta como fuente de $\mathrm{CO}_{2}$ hacia la atmósfera. Se ha estimado que en el canal de Ballenas la $\mathrm{pCO}_{2}$ puede alcanzar valores de aproximadamente $1000 \mu \mathrm{atm}$ (Hernández-Ayón et al. 2007b). Valores de $\mathrm{pCO}_{2}$ tan elevados como los encontrados en el canal de Ballenas se han registrado en zonas como las costas de Perú durante la época de surgencias (Friederich et al. 2008).

El enriquecimiento de CID antes descrito se ha atribuido principalmente a factores como la poca o nula estratificación (valores de $\phi$ bajos) ocasionada por las fuertes corrientes de marea (López y García 2003; Torres-Delgado et al. 2013, este número). También se le ha relacionado con el estado de mareas, que tiene efectos directos en los flujos de $\mathrm{CO}_{2}$ hacia la atmósfera en la RGI (Hidalgo-González et al. 1997). Por ejemplo, se ha encontrado que el mínimo de estratificación ocurre entre dos y tres días después de las mareas vivas (Simpson y Bowers 1981). El enriquecimiento de CID (así como de nutrientes y otras propiedades) en las aguas superficiales del canal de Ballenas se puede explicar con un mecanismo adicional a la mezcla por mareas; este mecanismo consiste en una surgencia de agua profunda producto de la convergencia de los flujos de ASS, que ingresan desde el sur por el umbral de San Lorenzo y desde el norte por el umbral del canal de Ballenas (López et al. 2006, Marinone 2007). Marinone (2007) sugirió que esta surgencia es intensa y está presente todo el año.

Las evidencias encontradas en este estudio revelan el papel del ASS como fuente de CID para el AGC cuando incrementa su volumen y se mezcla con el AGC por efecto de las mareas; pero, en la superficie, la concentración de CID también dependerá de los procesos biogeoquímicos que ahí 
transported from $\sim 200 \mathrm{~m}$, as can be observed in the temperature, salinity, density, and DIC profiles (figs. 5, 6). This subregion comprised the area of the sills located immediately to the south of the islands (fig. 1). It has been reported that a hydraulic jump originates in the sills, causing the SSW to transport its physicochemical properties directly towards the surface (Delgadillo-Hinojosa et al. 2001); this trasport could also result from the convergence of SSW flows (López et al. 2008). Moreover, this subregion is characterized by presenting the lowest $\phi$ values, resulting in DIC-rich water and low temperatures, salinities, and densities.

The temperature and salinity values recorded for GCW-2 are similar to those obtained by Godínez-Sandoval et al. (2003) for water samples collected in March in the northern $\mathrm{GC}$, which ranged from 14.8 to $18.0^{\circ} \mathrm{C}$ and from 35.2 to 36.2 , respectively. The northern GC is shallow $(250 \mathrm{~m}$ on average), so the evaporation process is more intense and leads to the formation of GCW in winter (Lavín et al. 1995). The water in this subregion presented low DIC concentrations in comparison with those found in Ballenas Channel, probably because GCW-2, which originated in the northern GC, was affected by photosynthesis and degasification processes.

GCW-3, found to the southeast of the MIR (figs. 2, 3, 4), was more stratified and presented a better defined thermocline and pycnocline (figs. 3, 5, 6), with $\phi$ values greater than $\sim 30 \mathrm{~J} \mathrm{~m}^{-3}$. This subregion was located above the San Pedro Mártir basin (station 38, fig. 4) where a permanent anticyclonic eddy has been observed (Marinone-Moschetto and Lavín-Peregrina 2003). It seems that part of the water that rises over the San Esteban and San Lorenzo sills flows southward along the surface because of the thermohaline circulation (Bray 1988, Delgadillo-Hinojosa 2000, Torres-Valdés 2000). On route to the surface, this water becomes warmer and therefore stratified, which in addition to the high nutrient content, favors photosynthesis and, consequently, carbon removal. Gaxiola-Castro et al. (1995) found an association between wintertime primary productivity and stratification in the GC. They also recorded higher primary productivity values to the south of the MIR and found low productivity in the areas of the MIR were strong mixing occurs. Moreover, diatoms and dinoflagellates dominated $(70 \%)$ at the MIR stations, whereas outside the MIR, flagellates and coccolithophorids were the dominant phytoplankton. This information gives an idea of the different responses of phytoplanktonic organisms to environmental conditions. In the first case, where dinoflagellates dominate, the water is rich in DIC and $\mathrm{pH}$ values are $\sim 7.7$, whereas GCW-3 has $\mathrm{pH}$ values $>8$ (Hernández-Ayón et al. 2007b).

\section{Relationship between DIC and temperature}

Based on data from the three Umbrales campaigns, there was a negative relationship between DIC and temperature $\left(r^{2}=0.93, P \leq 0.05, n=562\right)$ (fig. $\left.8 \mathrm{a}\right)$. Other variables of the ocurren. El AGC presentó tres tipos de agua que formaron tres zonas hidrográficamente distintas, con características particulares de temperatura, salinidad y CID que estuvieron, en mayor o menor medida, relacionadas con el ASS y otros procesos que a continuación se discuten.

La AGC-1, ubicada en el canal de Ballenas y al sur de las islas, presentó la mayor concentración de CID. En está subregión se presentan las corrientes de marea más intensas (Marinone-Moschetto y Lavín-Peregrina 2003), y el agua proviene de aproximadamente $200 \mathrm{~m}$ de profundidad, como se puede observar en los perfiles de temperatura, salinidad, densidad y CID (figs. 5, 6). La AGC-1 comprendió la zona de los umbrales localizados inmediatamente al sur de las islas (fig. 1). Se ha registrado que en los umbrales se origina un salto hidráulico que provoca que el ASS transporte sus propiedades físicoquímicas directamente hacia la superficie (Delgadillo-Hinojosa et al. 2001); este transporte también podría ser producto de la convergencia de los flujos de ASS (López et al. 2008). Además, esta subregión se caracteriza por presentar los valores más bajos de $\phi$, lo que resulta en temperaturas, salinidades y densidades bajas y en agua rica en CID.

La AGC-2 presentó valores de temperatura y salinidad similares a los registradas por Godínez-Sandoval et al. (2003) para muestras de agua recolectadas en marzo en el norte del GC. Dichos autores registraron valores de temperatura y salinidad que fluctuaron entre 14.8 y $18.0^{\circ} \mathrm{C}$ y entre 35.2 y 36.2 , respectivamente. El norte del GC es somero ( $250 \mathrm{~m}$ en promedio), por lo que los procesos de evaporación son más intensos y dan lugar a la formación de AGC en invierno (Lavín et al. 1995). Además, el agua de esta subregión presentó bajas concentraciones de CID en comparación con lo encontrado en el canal de Ballenas, posiblemente porque la AGC-2, que se origina en el norte del GC, ha sido afectada por procesos de desgasificación y fotosíntesis.

El agua de la AGC-3, que se localizó al sureste de la RGI (figs. 2, 3, 4), se encontró más estratificada y presentó una termoclina y una picnoclina mejor definidas (figs. 3, 5, 6), con valores de $\phi$ mayores que $\sim 30 \mathrm{~J} \mathrm{~m}^{-3}$. Esta subregión se localizó sobre la cuenca de San Pedro Mártir (estación 38, fig. 4) en donde se ha ubicado un giro anticiclónico permanente (Marinone-Moschetto y Lavín-Peregrina 2003). Creemos que parte del agua que surge de los umbrales San Esteban y San Lorenzo viaja por la superficie hacia el sur como resultado de la circulación termohalina (Bray 1988, Delgadillo-Hinojosa 2000, Torres-Valdés 2000). En su camino hacia la superficie dicha agua se calienta y, por lo tanto, se estratifica; esto, aunado a un alto contenido de nutrientes, favorece la fotosíntesis y, en consecuencia, la remoción de carbono. En un estudio realizado en invierno en el GC, Gaxiola-Castro et al. (1995) encontraron una asociación entre la productividad primaria y la estratificación. Estos autores también registraron valores más altos de productividad primaria hacia el sur de la RGI y encontraron que había baja productividad en las zonas de alta mezcla de la RGI. 
$\mathrm{CO}_{2}$ system, such as $\mathrm{pH}$ and $\mathrm{pCO}_{2}$, correlated with temperature. Mixing was found to control DIC distribution in the water column, but in the case of surface waters, the inverse relationship between DIC and temperature may be due to the fact that in the euphotic zone solar radiation warms the water and photosynthesis consumes $\mathrm{CO}_{2}$ causing a decrease in DIC. These processes result from the reaction $\mathrm{HCO}_{3}{ }^{-} \rightarrow \mathrm{CO}_{2}+$ $\mathrm{OH}^{-}$(Zirino et al. 1997) or the consumption of dissolved $\mathrm{CO}_{2}$.

We propose that in the MIR, both the surface and vertical wintertime concentrations of DIC are strongly controlled by physical processes. Specifically, surface carbon enrichment is favored by a greater presence of SSW in the water column in combination with strong mixing. Nonetheless, as mentioned previously, based on that observed in Ballenas Channel, there is marked temporal variation in the MIR (fig. 7). The evidence indicates that the SSW-mixing (enrichment) combination gradually disappears towards summertime because of (1) the sinking of SSW, resulting in a gradual decrease of DIC-rich water, and (2) the seasonal increase in the surface layer of GCW that becomes warmer and stratified, creating optimum conditions for phytoplankton growth. Hence, DIC

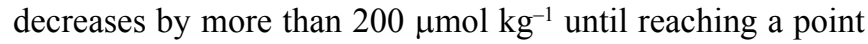
of equilibrium and the seasonal cycle starts again. DIC was maximum in winter and gradually decreased to values of $\sim 2080 \mu \mathrm{mol} \mathrm{kg}{ }^{-1}$ in summer. Equilibrium of surface waters likely occurs in autumn when they become oligotrophic, DIC enrichment initiating again when SSW rises in winter.

\section{Applications of the DIC vs temperature relationship}

It seems quite clear that DIC enrichment in late winter is caused by intense tidal mixing of SSW and GCW and that it gradually decreases towards summertime as SSW sinks and GCW dominates the surface layer. This oceanographic scenario allows the MIR to be viewed as a natural and special laboratory, and to a certain extent simplifies its study, assessment, and quantification.

For example, the DIC vs temperature relationship can also be applied when analyzing satellite data. Temperature imagery of $1-\mathrm{km}$ resolution can be used to map DIC estimates (fig. 8b, c) and indeed other variables of the carbon system, such as $\mathrm{pH}, \mathrm{pCO}_{2}$, and surface Omega aragonite. The temperature image for the first $11 \mathrm{~mm}$ recorded by the Modis Terra sensor on 21 March 2002 (one day before the March sampling) is shown in figure $8 \mathrm{~b}$. The oceanographic subregions referred to in this work can be discerned, with cold temperatures $\left(\sim 15^{\circ} \mathrm{C}\right)$ in the Ballenas Channel subregion (GCW-1), temperatures of 16 to $18^{\circ} \mathrm{C}$ towards the Sonora coastline (GCW-2), and temperatures of $>19^{\circ} \mathrm{C}$ for GCW-3. Figure $8 \mathrm{c}$ shows DIC calculated after applying the algorithm generated by the DIC-temperature relationship using measurements from the three campaigns. As expected, DIC values were highest in GCW-1, similar to those measured $\left.(\sim 2300 \mu \mathrm{mol} \mathrm{kg})^{-1}\right)$, and decreased towards the other two
Además, en la RGI se encontró un dominio de diatomeas y dinoflagelados en aproximadamente $70 \%$, mientras que fuera de la RGI predominaron los flagelados y los cocolitofóridos. Esta información, de alguna manera, da una idea de las diferentes respuestas de los organismos fitoplanctónicos frente a las condiciones del ambiente. En el primero caso, en donde dominan los dinoflagelados, el agua es rica en CID y el $\mathrm{pH}$ puede llegar a valores de $\sim 7.7$, mientras que en AGC-3, el agua alcanza valores de $\mathrm{pH}>8$ (Hernández-Ayón et al. 2007b).

\section{Relación CID vs temperatura}

Con base en los datos de las tres campañas, se encontró una relación negativa alta $\left(r^{2}=0.93, P \leq 0.05, n=562\right)$ entre el CID y la temperatura (fig. 8a). También otras variables del sistema del $\mathrm{CO}_{2}$, como el $\mathrm{pH}$ y la $\mathrm{pCO}_{2}$, se correlacionaron con la temperatura. Se encontró que la mezcla controla la distribución del CID en la columna de agua, pero en el caso de las aguas superficiales, la relación inversa entre el CID y la temperatura posiblemente se deba a que en la zona eufótica la radiación solar calienta el agua y la fotosíntesis utiliza el $\mathrm{CO}_{2}$, resultando en una disminución de CID. Estos procesos tendrían lugar mediante la reacción $\mathrm{HCO}_{3}^{-} \rightarrow \mathrm{CO}_{2}+\mathrm{OH}^{-}$ (Zirino et al. 1997) o por el simple consumo de $\mathrm{CO}_{2}$ disuelto.

En particular, proponemos que las concentraciones de CID en la RGI, tanto en la superficie como en la vertical, están fuertemente controladas, en invierno, por los procesos físicos. Específicamente, el enriquecimiento superficial de carbono está favorecido por una mayor presencia del ASS en la columna de agua en combinación con la fuerte mezcla. Sin embargo, como se mencionó anteriormente, en la RGI ocurre una clara variación temporal, tomando como referencia lo observado en el canal de Ballenas (fig. 7). Se presentaron evidencias de las cuales se interpreta que la combinación ASS-mezcla (enriquecimientos) va desapareciendo gradualmente hacia el verano por (1) el hundimiento de la ASS, que trae como resultado una disminución gradual de agua rica en CID, y (2) el aumento temporal del volumen de AGC en la superficie, que se va calentando y estratificando y va generando un ambiente óptimo para el crecimiento fitoplanctónico. Por consiguiente, el CID disminuye en más de $200 \mu \mathrm{mol} \mathrm{kg}^{-1}$ hasta llegar a un punto de equilibrio para que el ciclo estacional vuelva a empezar. Se observó un máximo de CID en invierno y una disminución gradual hasta llegar a valores de $\sim 2080 \mu \mathrm{mol} \mathrm{kg}^{-1}$ en el verano. Es probable que el otoño sea la época de equilibrio de las aguas superficiales porque alcanzan una fase oligotrófica, y el enriquecimiento de CID inicia de nuevo una vez que el ASS regresa a las aguas superficiales en invierno.

\section{Aplicaciones de la relación DIC vs temperatura}

Queda claro que el enriquecimiento de CID de finales de invierno fue producto del efecto de la mezcla intensa de ASS 

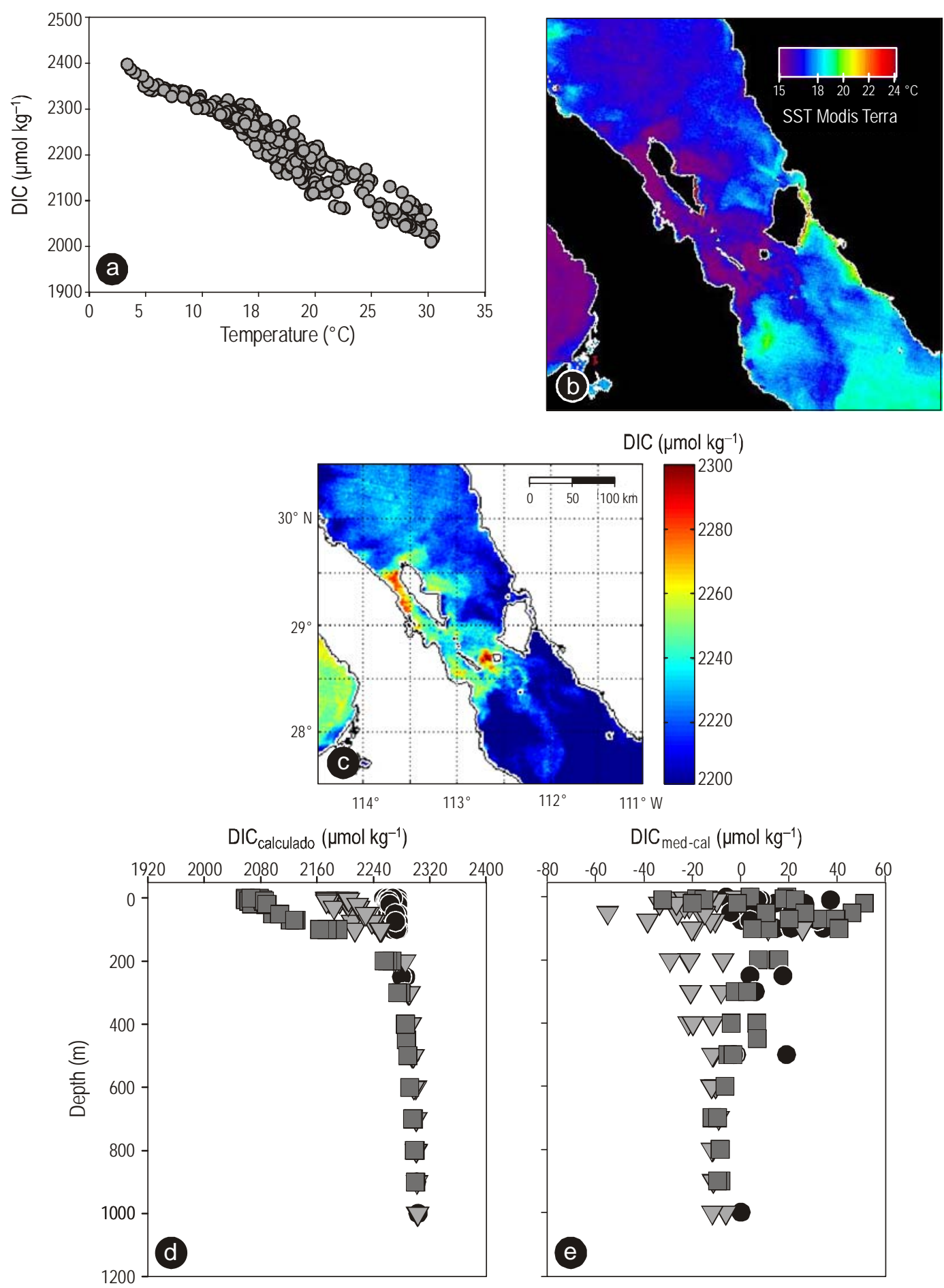

Figure 8. (a) Dissolved inorganic carbon (DIC) vs temperature relationship. Data were obtained from stations in the Midriff Islands region in winter 2002, spring 2003, and summer 2004. The figure includes two applications: (1) using 1-km resolution satellite temperature data (b), a DIC map (c) was generated using an equation; (2) using temperature data, DIC profiles were developed for winter $(\bullet)$, spring ( $\mathbf{\nabla})$ and summer ( $\mathbf{\square})$ conditions in Ballenas Channel. The DIC spatial distribution predicted by the model (d) and that measured (e) in the channel region was similar in both applications.

Figura 8. (a) Relación carbono inorgánico disuelto (DIC) vs temperatura. Los datos se obtuvieron de estaciones en la región de las grandes islas en invierno de 2002, primavera de 2003 y verano de 2004. La figura incluye dos aplicaciones: (1) a partir de datos satelitales de temperatura de $1 \mathrm{~km}$ de resolución (b), se elaboró un mapa de DIC (c) con una ecuación; (2) a partir de datos de temperatura, se generaron perfiles para condiciones de invierno $(\bullet)$, primavera $(\boldsymbol{\nabla})$ y verano $(\boldsymbol{\square})$ en el canal de Ballenas. La distribución espacial de DIC predicha por el modelo (d) y medida (e) en la región del canal fue similar en ambas aplicaciones. 
subregions. Another application of the DIC vs temperature relationship is in the simulation shown in figure 8d, obtained from the equation generated from the DIC-temperature relationship, for which data gathered during the three campaigns to the MIR were used. DIC was calculated using the Ballenas Channel temperature profiles for the three seasons. Figure 8 shows the profiles obtained from the simulation and one more that shows the differences between the measured and calculated DIC in order to assess this application (fig. 8d, e). The calculated profiles are very similar to those measured in the different seasons and the DIC ranges are also similar; however, it is possible to observe positive and negative differences in the first $100 \mathrm{~m}$ and how these differences decrease with depth.

The $\mathrm{pH}-\mathrm{DIC}$ correlation $\left(\mathrm{pH}, \mathrm{pCO}_{2}\right)$ can be useful when algorithms relating DIC content are developed using satellite imagery, continuous flux data, and hydrographic data for these types of sites, where physical processes facilitate the use of this application. Accurate satellite temperature data have been available for decades but progress has been made in recent years regarding the resolution of satellite measurements of the ocean. The MIR allows us to apply studies of this kind and could function as a calibration station for future satellites gathering spatial measurements of $\mathrm{CO}_{2}$. In this exercise, the average error of DIC calculated for every temperature datum in surface waters was $\pm 20 \mu \mathrm{mol} \mathrm{kg}{ }^{-1}$, which in terms of $\mathrm{pH}$ and $\mathrm{pCO}_{2}$ corresponds to $\sim 0.02$ units and $\sim 20 \mu \mathrm{atm}$, respectively. The differences in surface waters $(<100 \mathrm{~m})$ can be attributed to other processes because the equation-generated profile assumes a domain due to physical processes as was the case in March. The equation used here requires further assessment and is therefore not included. However, in view of its special oceanographic conditions, Ballenas Channel would be the first option to test algorithms in in situ studies of the $\mathrm{CO}_{2}$ system variables and apply them using satellite measurements.

\section{CONCLUSIONS}

The water masses identified in March 2002 were GCW, SSW, PIW, and PDW. The most predominant water masses (more than $80 \%$ of the total volume) in the MIR were GCW and SSW. GCW was found throughout the area in the first $100 \mathrm{~m}$. SSW was observed to the northwest, filling the Delfín, Salsipuedes, and Tiburón basins, and to the southeast, between 150 and $350 \mathrm{~m}$ depth. PIW did not cross the sills and was located to the southeast of the MIR, below $500 \mathrm{~m}$ depth. SSW was found to be a source of DIC to surface waters in the MIR, and when it increases in volume and mixes with GCW due to tidal effects, all the MIR becomes enriched with DIC. The seasonal analysis of Ballenas Channel showed that DIC enrichment decreases towards summertime when SSW sinks and the volume of GCW increases in the surface layer. In March, GCW presented three water types forming three oceanographic subregions associated with mixing processes con el AGC y que este enriquecimiento disminuye de manera gradual hacia el verano en la medida en que el ASS se va hundiendo y el AGC domina en la superficie. El escenario oceanográfico anterior invita a ver a la RGI como un laboratorio natural y especial, y simplifica en cierta medida su estudio, evaluación y cuantificación.

La relación CID vs temperatura puede aplicarse en estudios con datos satelitales. En la actualidad se pueden utilizar imágenes de satélite de temperatura con resolución de $1 \mathrm{~km}$ y obtener mapas de estimaciones de CID (fig. 8b, c) y también de otras variables del sistema de carbono, tales como $\mathrm{pH}$, $\mathrm{pCO}_{2}$ u Omega aragonita superficial. En la figura $8 \mathrm{~b}$ se muestra la imagen de temperatura satelital obtenida del sensor Modis Terra para los primeros $11 \mathrm{~mm}$ de profundidad el 21 de marzo de 2002 (un día antes del muestreo de marzo). En esta figura se pueden apreciar las subregiones oceanográficas referidas en este trabajo, siendo evidentes las bajas temperaturas $\left(\sim 15^{\circ} \mathrm{C}\right)$ que se presentan en esta época en la subregión del canal de Ballenas (AGC-1), las temperaturas de entre 16 y $18{ }^{\circ} \mathrm{C}$ hacia las costas de Sonora (AGC-2) y las temperaturas mayores que $19^{\circ} \mathrm{C}$ en la subregión AGC-3. Por otro lado, la figura 8c muestra el CID calculado después de aplicar el algoritmo generado por la relación CID vs temperatura usando las mediciones de las tres campañas. Tal como se esperaba, el mapa de CID muestra que los valores fueron más altos en AGC-1, similares a los medidos $\left(2300 \mu \mathrm{mol} \mathrm{kg}{ }^{-1}\right)$, y disminuyeron hacia las otras dos subregiones. Una segunda aplicación de la relación CID vs temperatura es en la simulación que se muestra en la figura 8d, obtenida de la ecuación generada a partir de la relación CID vs temperatura, para la cual se utilizaron los datos generados de los tres cruceros realizados en la RGI. Mediante este ejercicio se obtuvo un CID calculado usando los perfiles de temperatura del canal de Ballenas de las tres épocas del año. En la figura 8 se muestran los perfiles obtenidos de la simulación y uno más que muestra las diferencias entre lo medido y lo calculado con el propósito de evaluar esta aplicación (fig. 8d, e). Se puede notar que los perfiles calculados son muy parecidos a los perfiles medidos en las diferentes épocas del año y que los rangos de CID también son similares. Sin embargo, se pueden apreciar diferencias positivas y negativas en los primeros $100 \mathrm{~m}$ y cómo estas diferencias disminuyen con la profundidad.

La correlación $\mathrm{pH}-\mathrm{CID}\left(\mathrm{pH}, \mathrm{pCO}_{2}\right)$ puede ser útil en el desarrollo de algoritmos que relacionen el contenido de CID a partir de imágenes de satélite, datos de flujo continuo y datos hidrográficos en este tipo de sitios, donde los procesos físicos facilitan el uso de dicha aplicación. Por décadas han estado disponibles datos de satélite de mediciones precisas de temperatura, pero recientemente se han realizado progresos en la resolución de mediciones del océano usando satélites. La RGI nos permite realizar estudios de este tipo y podría funcionar como una estación de calibración para futuros satélites que inicien mediciones espaciales de $\mathrm{CO}_{2}$. En este ejercicio, el error promedio de CID calculado por cada dato de temperatura en las aguas superficiales fue de $\pm 20 \mu \mathrm{mol} \mathrm{kg}^{-1}$, el cual 
both at the surface and vertically: (a) GCW-1 was the coldest and least stratified, and had high DIC content; (b) GCW-2 was more saline and had less DIC content than GCW-1, and its chemical composition was possibly influenced by the northern GC; and (c) GCW-3 was the warmest, less dense, and most stratified, and had the lowest DIC content. The DIC vs temperature relationship is a good tool that could be applied in future studies using satellite imagery.

\section{ACKNOWLEDGMENTS}

We acknowledge funding from the Mexican Council for Science and Technology (CONACYT) to project "Umbrales G33464-T" and from the Autonomous University of Baja California (UABC, project No. 615). We thank Tania Camiro-Vargas for her enthusiastic participation on the 2003 cruise, and Vanessa Torres-Delgado and the reviewers for their valuable comments and suggestions.

English translation by Christine Harris.

\section{REFERENCES}

Álvarez-Borrego S, Schwartzlose RA. 1979. Water masses of the Gulf of California. Cienc. Mar. 6: 43-63.

Álvarez-Borrego S, Rivera JA, Gaxiola-Castro G, Acosta-Ruiz MJ, Schwartzlose R. 1978. Nutrientes en el Golfo de California. Cienc. Mar. 5: 53-71.

Argote ML, Amador A, Lavín M. 1995. Tidal dissipation and stratification in the Gulf of California. J. Geophys. Res. 100(C8): 16103-16118.

Badan-Dangon A, Hendershott MC, Lavín MF. 1991. Underway Doppler current profiles in the Gulf of California. EOS Trans. AGU 72(209): 217-218.

Bray N. 1988. Thermohaline circulation in the Gulf of California. J. Geophys. Res. 93(C5): 4993-5020.

Castro R, Mascarenhas AS, Durazo R, Collins CA. 2000. Variación estacional de la temperatura y salinidad en la entrada del golfo de California, México. Cienc. Mar. 26: 561-583 pp.

Delgadillo-Hinojosa F. 2000. Biogeoquímica del cadmio y manganeso en el golfo de California. PhD thesis, Universidad Autónoma de Baja California, Ensenada, México, 183 pp.

Delgadillo-Hinojosa F, Macías-Zamora JV, Segovia-Zavala JA, Torres-Valdés S. 2001. Cadmiun enrichment in the Gulf of California. Mar. Chem. 75: 109-122 pp.

Falkowski PG, Raven JA. 1997. Aquatic Photosynthesis. Blackwell Science, Oxford, UK, 375 pp.

Feely RA, Sabine CL, Hernández-Ayón JM, Ianson D, Hales B. 2008. Evidence for upwelling of corrosive "acidified" water onto the Continental Shelf. Science 320(5882): 1490-1492. http://dx.doi.org/10.1126/science.1155676

Flores-de-Santiago FJ, Santamaría-del-Ángel E, González-Silvera A, Martínez-Díaz-de-León A, Millán-Núñez R, Kovacs JM. 2007. Assessing dynamics micro-regions in the Great Islands of the Gulf of California based on MODIS aqua imagery products. Proc. Society of Photo-Optical Instrumentation Engineers 6680. http://dx.doi.org/10.1117/12.732574

Friederich GE, Ledesma J, Ulloa O, Chávez FP. 2008. Air-sea carbon dioxide fluxes in the coastal southeastern tropical Pacific. Prog. Oceanogr. 79: 156-166. en términos de $\mathrm{pH}$ y $\mathrm{pCO}_{2}$ corresponde a $\sim 0.02$ unidades $\mathrm{y}$ $\sim 20 \mu \mathrm{atm}$, respectivamente. Las diferencias en las aguas superficiales $(<100 \mathrm{~m})$ se pueden atribuir a otros procesos, ya que el perfil generado de la ecuación supone un dominio debido a procesos físicos como fue el caso de marzo. La ecuación utilizada en este trabajo aún tendría que ser evaluada con más mediciones y es por eso que no se incluye en este trabajo. Sin embargo, por sus condiciones oceanográficas especiales, el canal de Ballenas sería la primera opción para probar algoritmos vía estudios in situ de variables del sistema del $\mathrm{CO}_{2}$ y aplicarlos usando mediciones satelitales.

\section{CONCLUSIONES}

Las masas de agua identificadas durante marzo de 2002 fueron AGC, ASS, AIP y APP. Las masas de agua predominantes en la RGI fueron el AGC y ASS, que corresponden a más del $80 \%$ del total del volumen. El AGC se encontró en toda la superficie en los primeros $100 \mathrm{~m}$. El ASS tuvo dos distribuciones distintas: al noroeste se encontró llenando las cuencas Delfín, Salsipuedes y Tiburón, y al sureste se encontró entre 150 y $350 \mathrm{~m}$ de profundidad. El AIP no atravesó los umbrales y se localizó al sureste de la RGI, por debajo de los $500 \mathrm{~m}$. Se encontró que el ASS es fuente de CID en las aguas superficiales en la RGI, y al aumentar su volumen en invierno y al mezclarse con el AGC por efecto de las mareas, toda la RGI se enriquece con CID. El enriquecimiento disminuye hacia el verano, cuando el ASS se hunde y al mismo tiempo el volumen de AGC aumenta en la superficie, según el análisis temporal del canal de Ballenas. En marzo, el AGC presentó tres tipos de agua formando tres subregiones oceanográficas asociadas a los procesos de mezcla con el ASS, tanto en la superficie como en la vertical: (a) la AGC-1 fue más fría y presentó los valores más bajos de estratificación y una alta concentración de CID; (b) la AGC-2 fue más salina y presentó una concentración de CID menor que la AGC-1, y su composición química posiblemente fue modificada por la influencia del norte del GC; y (c) la AGC-3 fue más cálida y menos densa, y presentó mayor estratificación y las menores concentraciones de CID. Se propone que la relación CID vs temperatura es una buena herramienta que podría aplicarse en futuros estudios mediante imágenes de satélite.

\section{Agradecimientos}

Los autores agradecen el financiamiento por parte del Consejo Nacional de Ciencia y Tecnología (CONACYT) al proyecto "Umbrales G33464-T" y por parte de la Universidad Autónoma de Baja California (proyecto No. 615). También agradecemos a Tania Camiro-Vargas por su entusiasta participación en el crucero de 2003 y a Vanessa TorresDelgado y a los revisores de este trabajo por sus comentarios positivos y sugerencias. 
Gaxiola-Castro GS, Alvarez-Borrego, Shwartzlose RA. 1978. Sistema del bióxido de carbono en el Golfo de California. Cienc. Mar. 5: 25-40.

Gaxiola-Castro G, García-Cordova J, Valdez-Holguín JE, BotelloRuvalcaba M. 1995. Spatial distribution of chlorophyll $a$ and primary productivity in relation to winter physical structure in the Gulf of California. Cont. Shelf. Res. 15: 1043-1059.

Godínez-Sandoval VM, Lavín M, Álvarez-Sánchez LG, SánchezMancilla S, Ramírez-Mendoza R. 2003. Datos hidrográficos de la campaña oceanográfica: Alto Golfo de California FU0002: del 24 de febrero al 1 de marzo del 2000. Informe técnico 2332. Comunicaciones Académicas, Serie Oceanografía Física, CICESE, $110 \mathrm{pp}$.

Hernández-Ayón JM, Belli SL, Zirino A 1999. pH, alkalinity and total $\mathrm{CO}_{2}$ in coastal seawater by potentiometric titration with a difference derivative readout. Anal. Chim. Acta. 394: 101-108.

Hernández-Ayón JM, Zirino-Weiss A, Delgadillo-Hinojosa F, Galindo-Bect S. 2007a. Procesos de control del carbono inorgánico disuelto en el golfo de California en condiciones de verano. Capítulo III. Procesos biogeoquímicos del carbono. In: Carbono en Ecosistemas Acuáticos de México. Instituto Nacional de Ecología y Centro de Investigación Científica y de Educación Superior de Ensenada. ISBN: 978-968-817-855-3.

Hernández-Ayón JM, Delgadillo-Hinojosa F, Camiro-Vargas T, López-Mariscal M. 2007b. Estudio de las variables del $\mathrm{CO}_{2}$ en la región de las grandes islas en el golfo de California en condiciones de invierno. Capítulo IV. $\mathrm{CO}_{2}$ en aguas costeras y lacustres. In: Carbono En Ecosistemas Acuáticos de México. Instituto Nacional de Ecología y Centro de Investigación Científica y de Educación Superior de Ensenada. ISBN: 978968-817-855-3.

Hidalgo-González R, Álvarez-Borrego S, Zirino A. 1997. Mezcla en la región de las grandes islas del golfo de California: Efecto de la $\mathrm{pCO}_{2}$ superficial. Cienc. Mar. 23: 317-327.

Lavín MS, Gaxiola-Castro G, Robles JM. 1995. Winter water masses and nutrients in the northern Gulf of California. J. Geophys. Res. 100(C5): 8587-8605.

Linacre L, Durazo R, Hernández-Ayón JM, Cervantes-Díaz G, Delgadillo-Hinojosa F, Lara-Lara JR, Camacho-Ibar V, Siqueiros-Valencia A, Bazán-Guzmán C. 2010. Temporal variability of the physicochemical water characteristics at a coastal monitoring observatory: Station ENSENADA. Cont. Shelf Res. 30: 1730-1742.

López M, García J. 2003. Moored observations in the northern Gulf of California: A strong bottom current. J. Geophys. Res. 108(C2, 3048): 1-18. http://dx.doi.org/10.1029/2002JC001492

López M, Candela J, Argote ML. 2006. Why does the Ballenas Channel have the coldest SST in the Gulf of California? Geophy. Res.Lett. 33, L11603. http://dx.doi.org/10.1029/2006GL025908

López M, Candela J, García J. 2008. Two overflows in the Northern Gulf of California. J. Geophys. Res. 113(C8).

http://dx.doi.org/10.1029/2007JC004575
Marinone SG. 2007. A note on "Why does the Ballenas Channel have the coldest SST in the Gulf of California?". Geophys. Res. Lett. 34, L02607. http://dx.doi.org/10.1029/2006GL028589

Marinone-Moschetto SGL, Lavín-Peregrina MF. 2003. Residual flow and mixing in the large islands region of the central Gulf of California. In: Velasco Fuentes OU, Sheinbaum J, Ochoa J (eds.), Nonlinear Processes in Geophysical Fluid Dynamics. Kluwer Academic Publishers, pp. 213-236.

Martínez-Díaz-de-León A, Pacheco-Ruíz I, Delgadillo-Hinojosa F, Zertuche-González JA, Chee-Barragán A, Blanco-Betancourt R, Guzmán-Calderón JM, Gálvez-Telles A. 2006. Spatial and temporal variability of the sea surface temperature in the Ballenas-Salsipuedes Channel (central Gulf of California). J. Geophys. Res. 11, C02008.

Paden CA, Winant CD, Abbott MR. 1993. Tidal and atmospheric forcing of the upper ocean in the Gulf of California. 2. Surface heat flux. J. Geophys. Res. 98(C11): 20091-20103.

Ribas-Ribas M, Hernández-Ayón JM, Camacho-Ibar VF, CabelloPasini A, Mejía-Trejo A, Durazo R, Galindo-Bect S, Souza AJ, Forja JM, Siqueiros-Valencia A. 2011. Effects of upwelling, tides and biological processes on the inorganic carbon system of a coastal lagoon in Baja California. Estuar. Coast. Shelf Sci. 95(4): 367-376.

Simpson JH, Bowers D. 1981. Models of stratification and frontal movement in shelf seas. Deep-Sea Res. 28A(7): 727-738.

Soto-Mardones L, Marinone SG, Parés-Sierra A. 1999. Variabilidad espaciotemporal de la temperatura superficial del mar en el golfo de California. Cienc. Mar. 25: 1-30.

Santamaria-del-Angel E, Alvarez-Borrego S, Muller-Karger FE. 1994. Gulf of California biogeographic regions based on coastal zone color scanner imagery. J. Geophys. Res. 99(C4): 7411-7421.

Torres-Delgado EV, Delgadillo-Hinojosa F, Camacho-Ibar VF, Huerta-Díaz MA, Segovia-Zavala JA, Hernández-Ayón JM, Galindo-Bect S. 2013. Wintertime enrichment of inorganic nutrients in the Ballenas Channel, Gulf of California. Cienc. Mar. 39: 47-64.

http://dx.doi.org/10.7773/cm.v39i2.2236

Torres-Orozco E. 1993. Análisis volumétrico de las masas de agua del golfo de California. MSc thesis, Centro de Investigación científica y de Educación Superior de Ensenada, México, 80 pp.

Torres-Valdés S. 2000. Distribución espacial y variabilidad temporal de nutrientes en el golfo de California. MSc thesis, Universidad Autónoma de Baja California, Ensenada, México, 62 pp.

Zirino A, Hernández-Ayón JM, Fuhrman RA, Álvarez-Borrego S, Gaxiola-Castro G, Lara-Lara R, Bernstein RL. 1997. Estimate of surface $\mathrm{PCO}_{2}$ in the Gulf of California from underway $\mathrm{pH}$ measurements and satellite imagery. Cienc. Mar. 23: 1-22.

Received October 2012, received in revised form April 2013, accepted April 2013. 